Canad. J. Math. Vol. 71 (1), 2019 pp. 213-246

http://dx.doi.org/10.4153/CJM-2018-022-7

(c) Canadian Mathematical Society 2018

\title{
On an Enriques Surface Associated With a Quartic Hessian Surface
}

Dedicated to Professor Jonghae Keum on the occasion of his 60th birthday

Ichiro Shimada

\begin{abstract}
Let $Y$ be a complex Enriques surface whose universal cover $X$ is birational to a general quartic Hessian surface. Using the result on the automorphism group of $X$ due to Dolgachev and Keum, we obtain a finite presentation of the automorphism group of $Y$. The list of elliptic fibrations on $Y$ and the list of combinations of rational double points that can appear on a surface birational to $Y$ are presented. As an application, a set of generators of the automorphism group of the generic Enriques surface is calculated explicitly.
\end{abstract}

\section{Introduction}

We work over the complex number field $\mathbb{C}$. An involution on a $K 3$ surface is called an Enriques involution if it has no fixed-points. Let $\bar{X}$ be a general quartic Hessian surface, which means that $\bar{X}$ is the quartic surface in $\mathbb{P}^{3}$ defined by the equation

$$
\operatorname{det}\left(\frac{\partial^{2} F}{\partial x_{i} \partial x_{j}}\right)=0,
$$

where $F=F\left(x_{1}, \ldots, x_{4}\right)$ is a general cubic homogeneous polynomial. Then $\bar{X}$ has ten ordinary nodes $p_{\alpha}$ as its only singularities, and contains exactly ten lines $\ell_{\beta}$. Let $A$ denote the set of subsets $\alpha$ of $\{1,2,3,4,5\}$ with $|\alpha|=3$, and $B$ the set of subsets $\beta$ of $\{1, \ldots, 5\}$ with $|\beta|=2$. Then $p_{\alpha}$ and $\ell_{\beta}$ can be indexed by $\alpha \in A$ and $\beta \in B$, respectively, in such a way that $p_{\alpha} \in \ell_{\beta}$ if and only if $\alpha \supset \beta$. Let $X \rightarrow \bar{X}$ be the minimal resolution, let $E_{\alpha}$ be the exceptional curve over $p_{\alpha}$, and let $L_{\beta}$ be the strict transform of $\ell_{\beta}$. It is classically known [8] that the $K 3$ surface $X$ has an Enriques involution $\varepsilon$ that interchanges $E_{\alpha}$ and $L_{\bar{\alpha}}$ for each $\alpha \in A$, where $\bar{\alpha}:=\{1, \ldots, 5\} \backslash \alpha$. We denote the quotient morphism by $\pi: X \rightarrow Y:=X /\langle\varepsilon\rangle$.

The first application of Borcherds's method [3,4] to the automorphism group of $K 3$ surfaces was given by Kondo [15]. A set of generators of the automorphism group $\operatorname{Aut}(X)$ of the $K 3$ surface $X$ above was obtained in Dolgachev and Keum [8] by this method. On the other hand, we presented a computer algorithm for Borcherds's method [27]. Using this computational tool, we obtain an explicit description of

Received by the editors May 27, 2017; revised April 16, 2018.

Published electronically December 3, 2018.

This work was supported by JSPS KAKENHI Grant Number 16H03926 and 16K13749.

AMS subject classification: 14J28, 14Q10.

Keywords: Enriques surface, K3 surface, automorphism, lattice. 
$\operatorname{Aut}(X)$ and a fundamental domain $D_{X}$ of its action on the cone

$$
N(X):=\left\{x \in \mathcal{P}_{X} \mid\langle x,[C]\rangle \geq 0 \text { for all curves } C \text { on } X\right\}
$$

in $S_{X} \otimes \mathbb{R}$, where $S_{X}$ is the Néron-Severi lattice of $X$ with the intersection form $\langle\cdot, \cdot\rangle$, $\mathcal{P}_{X}$ is the connected component of $\left\{x \in S_{X} \otimes \mathbb{R} \mid\langle x, x\rangle>0\right\}$ containing an ample class, and $[C] \in S_{X}$ is the class of a curve $C \subset X$.

By analyzing this result, we obtain the following results on the automorphism group Aut $(Y)$ of the Enriques surface $Y$. Let $\iota_{\alpha}: X \rightarrow X$ denote the involution of $X$ induced by the double covering $\bar{X} \rightarrow \mathbb{P}^{2}$ obtained from the projection with the center $p_{\alpha} \in \bar{X}$. In [8], it was proved that $\iota_{\alpha}$ commutes with $\varepsilon$. Hence $\iota_{\alpha}$ induces an involution $j_{\alpha}: Y \rightarrow Y$ of $Y$.

Theorem 1.1 The automorphism group $\operatorname{Aut}(Y)$ of $Y$ is generated by the ten involutions $j_{\alpha}$. The following relations form a set of defining relations of $\operatorname{Aut}(Y)$ with respect to these generators $j_{\alpha}$ :

(i) $j_{\alpha}^{2}=$ id for each ordinary node $p_{\alpha}$;

(ii) $\left(j_{\alpha} j_{\alpha^{\prime}} j_{\alpha^{\prime \prime}}\right)^{2}=$ id for each triple $\left(p_{\alpha}, p_{\alpha^{\prime}}, p_{\alpha^{\prime \prime}}\right)$ of distinct three ordinary nodes such that there exists a line in $\bar{X}$ passing through $p_{\alpha}, p_{\alpha^{\prime}}, p_{\alpha^{\prime \prime}}$;

(iii) $\left(j_{\alpha} j_{\alpha^{\prime}}\right)^{2}=\mathrm{id}$ for each pair $\left(p_{\alpha}, p_{\alpha^{\prime}}\right)$ of distinct ordinary nodes such that the line in $\mathbb{P}^{3}$ passing through $p_{\alpha}$ and $p_{\alpha^{\prime}}$ is not contained in $\bar{X}$.

Remark 1.2 Recently Dolgachev [7] studied the group generated by the involutions $j_{\alpha}$ (that is, Aut $(Y)$ by Theorem 1.1), and showed that this group is isomorphic to a subgroup of $\Gamma \rtimes \mathfrak{S}_{5}$, where $\Gamma$ is a group isomorphic to the Coxeter group with the anti-Petersen graph as its Coxeter graph [7, Corollary 4.4]. (This corollary was also known to Mukai.)

Mukai and Ohashi informed us that they also proved, without computer-aided calculations, that $\operatorname{Aut}(Y)$ is generated by $j_{\alpha}(\alpha \in A)[21]$.

Let $S_{Y}$ denote the lattice of numerical equivalence classes of divisors on $Y$, which is isomorphic to $H^{2}(Y, \mathbb{Z}) /($ torsion) equipped with the cup product. By the result of $[14,19,20]$, we know that the action of $\operatorname{Aut}(Y)$ on $S_{Y}$ is faithful. Theorem 1.1 is proved by investigating this faithful action. More precisely, let $\mathcal{P}_{Y}$ denote the connected component of $\left\{y \in S_{Y} \otimes \mathbb{R} \mid\langle y, y\rangle>0\right\}$ containing an ample class. We put $N(Y):=\left\{y \in \mathcal{P}_{Y} \mid\langle y,[C]\rangle \geq 0\right.$ for all curves $C$ on $\left.Y\right\}$. It is obvious that $\operatorname{Aut}(Y)$ acts on $N(Y)$. We give a description of a fundamental domain $D_{Y}$ of the action of $\operatorname{Aut}(Y)$ on $N(Y)$. For $v \in S_{Y} \otimes \mathbb{R}$ with $\langle v, v\rangle<0$, let $(v)^{\perp}$ denote the hyperplane in $\mathcal{P}_{Y}$ defined by $\langle v, x\rangle=0$.

Theorem 1.3 There exists a fundamental domain $D_{Y}$ of the action of $\operatorname{Aut}(Y)$ on $N(Y)$ with the following properties.

(i) The fundamental domain $D_{Y}$ is bounded by $10+10$ hyperplanes $\left(\bar{u}_{\alpha}\right)^{\perp}$ and $\left(\bar{v}_{\alpha}\right)^{\perp}$, where $\alpha$ runs through the set $A$.

(ii) For each $\alpha \in A$, the vector $\bar{u}_{\alpha}$ is the class of the smooth rational curve $\pi\left(E_{\alpha}\right)=$ $\pi\left(L_{\bar{\alpha}}\right)$ on $Y$, and hence $\left(\bar{u}_{\alpha}\right)^{\perp}$ is a hyperplane bounding $N(Y)$. 


\begin{tabular}{cc}
{$\left[R_{\text {full }}, R_{\text {half }}\right]$} & number \\
\hline$\left[\varnothing, A_{4}\right]$ & 1 \\
{$\left[A_{5}+A_{1}, \varnothing\right]$} & 10 \\
{$\left[D_{5}, \varnothing\right]$} & 5 \\
{$\left[E_{6}, \varnothing\right]$} & 5
\end{tabular}

Table 1.1: Elliptic fibrations on $Y$

(iii) For each $\alpha \in A$, the involution $j_{\alpha} \in \operatorname{Aut}(Y)$ maps $D_{Y}$ to the chamber adjacent to $D_{Y}$ across the wall $D_{Y} \cap\left(\bar{v}_{\alpha}\right)^{\perp}$ of $D_{Y}$.

Let $Z$ be an Enriques surface. Then an elliptic fibration $\phi: Z \rightarrow \mathbb{P}^{1}$ has exactly two multiple fibers $2 E_{1}$ and $2 E_{2}$.

Theorem 1.4 Up to the action of Aut $(Y)$, the Enriques surface $Y$ has exactly $1+10+5+5$ elliptic fibrations. Their ADE-types of reducible fibers are given in Table 1.1, in which $R_{\text {full }}$ and $R_{\text {half }}$ denote the ADE-types of non-multiple reducible fibers and of the half of the multiple reducible fibers, respectively.

An RDP-configuration on an Enriques surface $Z$ is the exceptional divisor of a birational morphism $Z \rightarrow \bar{Z}$, where $\bar{Z}$ has only rational double points as its singularities. The support of an RDP-configuration is an ADE-configuration of smooth rational curves.

Theorem 1.5 Up to the action of $\operatorname{Aut}(Y)$, the Enriques surface $Y$ has exactly 750 non-empty RDP-configurations. Their ADE-types are given in Table 1.2.

Remark 1.6 All RDP-configurations on complex Enriques surfaces are classified by a lattice-theoretic equivalence relation [30].

The lattice $S_{Z}$ of numerical equivalence classes of divisors on an Enriques surface $Z$ is isomorphic to the even unimodular hyperbolic lattice $L_{10}$ of rank 10, which is unique up to isomorphism. The group $\mathrm{O}^{+}\left(L_{10}\right)$ of isometries of $L_{10}$ preserving a positive cone $\mathcal{P}_{10}$ of $L_{10} \otimes \mathbb{R}$ is generated by the reflections with respect to the roots. Vinberg [32] determined the shape of a standard fundamental domain of the action of $\mathrm{O}^{+}\left(L_{10}\right)$ on $\mathcal{P}_{10}$. (See Section 3.1.) Hence we call these fundamental domains Vinberg chambers.

Barth and Peters [2] determined the automorphism group $\operatorname{Aut}\left(Z_{\text {gen }}\right)$ of a generic Enriques surface $Z_{\text {gen }}$. (See also Nikulin [23].) Let $\mathcal{P}_{Z_{\text {gen }}}$ be the positive cone of $S_{Z_{\text {gen }}} \otimes \mathbb{R}$ containing an ample class. We identify $S_{Z_{\text {gen }}}$ with $L_{10}$ by an isometry that maps $\mathcal{P}_{Z_{\text {gen }}}$ to $\mathcal{P}_{10}$. Since $Z_{\text {gen }}$ contains no smooth rational curves, we have $N\left(Z_{\text {gen }}\right)=\mathcal{P}_{10}$. Note that the discriminant form $q_{L_{10}(2)}$ of $L_{10}(2)$ is a quadratic form 


\begin{tabular}{ccccc} 
ADE-type & number & & ADE-type & number \\
\cline { 2 - 3 } \cline { 4 - 5 }$E_{6}$ & 60 & & $A_{3}+A_{1}$ & 60 \\
$A_{5}+A_{1}$ & 60 & & $2 A_{2}$ & 15 \\
$3 A_{2}$ & 5 & & $A_{2}+2 A_{1}$ & 60 \\
$D_{5}$ & 60 & & $4 A_{1}$ & 5 \\
$A_{5}$ & 60 & & $A_{3}$ & 30 \\
$A_{4}+A_{1}$ & 60 & & $A_{2}+A_{1}$ & 60 \\
$A_{3}+2 A_{1}$ & 30 & & $3 A_{1}$ & 30 \\
$2 A_{2}+A_{1}$ & 30 & & $A_{2}$ & 15 \\
$D_{4}$ & 10 & & $2 A_{1}$ & 30 \\
$A_{4}$ & 60 & & $A_{1}$ & 10
\end{tabular}

Table 1.2: RDP-configurations on $Y$

over $\mathbb{F}_{2}$ with Witt defect 0 , and hence its automorphism group $\mathrm{O}\left(q_{L_{10}(2)}\right)$ is isomorphic to $\mathrm{GO}_{10}^{+}(2)$ in the notation of [1]. Moreover, the natural homomorphism

$$
\rho: \mathrm{O}^{+}\left(L_{10}\right) \rightarrow \mathrm{O}\left(q_{L_{10}(2)}\right) \cong \mathrm{GO}_{10}^{+}(2)
$$

is surjective. It was shown in [2] that the natural representation of $\operatorname{Aut}\left(Z_{\text {gen }}\right)$ on $S_{Z_{\text {gen }}} \cong L_{10}$ identifies $\operatorname{Aut}\left(Z_{\text {gen }}\right)$ with the kernel of $\rho$. In particular, $\operatorname{Aut}\left(Z_{\text {gen }}\right)$ is isomorphic to a normal subgroup of $\mathrm{O}^{+}\left(L_{10}\right)$ with index

$$
\left|\mathrm{GO}_{10}^{+}(2)\right|=2^{21} \cdot 3^{5} \cdot 5^{2} \cdot 7 \cdot 17 \cdot 31=46998591897600 .
$$

By the following theorem, we can describe the way the automorphism group changes in $\mathrm{O}^{+}\left(L_{10}\right)$ under the specialization from $Z_{\text {gen }}$ to $Y$ (see Remark 7.18).

Theorem 1.7 Under an isometry $S_{Y} \cong L_{10}$ that maps $\mathcal{P}_{Y}$ to $\mathcal{P}_{10}$, the fundamental domain $D_{Y}$ in Theorem 1.3 is a union of the following number of Vinberg chambers:

$$
2^{14} \cdot 3 \cdot 5 \cdot 7 \cdot 17 \cdot 31=906608640
$$

This theorem also gives us a method for calculating an explicit set of generators of $\operatorname{Aut}\left(Z_{\text {gen }}\right)$. Using this set, we carry out an experiment on the entropies of automorphisms of $Z_{\text {gen }}$.

The moduli of quartic Hessian surfaces has been studied by several authors in order to investigate the moduli of cubic surfaces $[6,13,16]$. Dardanelli and van Geemen [6] studied several interesting subfamilies of this moduli. It seems to be an interesting problem to investigate the change of the automorphism group under specializations of $Y$ to members of these subfamilies by the method given in this paper.

As was shown [21, Remark 4(2)], there exists a specialization from $Y$ to the Enriques surface $Y_{\mathrm{VI}}$ with $\operatorname{Aut}\left(Y_{\mathrm{VI}}\right) \cong \mathfrak{S}_{5}$ that appeared in the Nikulin-Kondo classification of Enriques surfaces with finite automorphism groups [14,23]. Kondo pointed out that the roots $\bar{u}_{\alpha}, \bar{v}_{\alpha}$ defining the walls of $D_{Y}$ given in Theorem 1.3 have the same configuration as the smooth rational curves on $Y_{\mathrm{VI}}, c f$. (7.1), (7.2), and [14, Figure 
6.4]. It is also an interesting problem to investigate the change of the automorphism group under various generalizations of the seven Enriques surfaces with finite automorphism groups.

The first application of Borcherds's method to the automorphism group of an Enriques surface was given in [29], in which we investigated an Enriques surface whose universal cover is a $K 3$ surface of Picard number 20 with the transcendental lattice of discriminant 36.

This paper is organized as follows. In Section 2, we collect preliminaries about lattices and chambers. In Section 3, we recall the results on the even unimodular hyperbolic lattices due to Vinberg [32] and Conway [5]. In Section 4, we explain Borcherds's method and its application to $K 3$ surfaces. In Section 5, we present some algorithms to study the geometry of an Enriques surface. In particular, we give an application of Borcherds's method to an Enriques surface. In Section 6, we re-calculate, by the algorithm in [27], the results of Dolgachev and Keum [8] on the general quartic Hessian surface, and convert these results into machine-friendly format. With these preparations, the main results are proved in Section 7. In the last section, we calculate a set of generators of $\operatorname{Aut}\left(Z_{\text {gen }}\right)$, and search for elements of $\operatorname{Aut}\left(Z_{\text {gen }}\right)$ with small entropies.

For the computation, we used GAP [10]. The computational data are presented in the author's webpage [31]. In fact, once the basis of the Leech lattice (Table 3.1), the basis of $S_{X}$ (6.3), the embedding $S_{X} \hookrightarrow L_{26}$ (Table 6.1), and the basis of $S_{Y}$ (Table 6.4) are fixed, the other data can be derived by the algorithms in this paper.

\section{Preliminaries}

\subsection{Lattices}

A submodule $M$ of a free $\mathbb{Z}$-module $L$ is said to be primitive if $L / M$ is torsion-free. A non-zero vector $v \in L$ is primitive if $\mathbb{Z} v \subset L$ is primitive.

A lattice is a free $\mathbb{Z}$-module $L$ of finite rank with a non-degenerate symmetric bilinear form $\langle\cdot, \cdot\rangle: L \times L \rightarrow \mathbb{Z}$. Let $m$ be a non-zero integer. For a lattice $(L,\langle\cdot, \cdot\rangle)$, we denote by $L(m)$ the lattice $(L, m\langle\cdot, \cdot\rangle)$. Every vector of $L \otimes \mathbb{R}$ is written as a row vector, and the orthogonal group $\mathrm{O}(L)$ of $L$ acts on $L$ from the right. We put

$$
L^{\vee}:=\operatorname{Hom}(L, \mathbb{Z}), \quad L_{\mathbb{Q}}:=L \otimes \mathbb{Q}, \quad L_{\mathbb{R}}:=L \otimes \mathbb{R} .
$$

Then we have natural inclusions $L \hookrightarrow L^{\vee} \hookrightarrow L_{\mathbb{Q}} \hookrightarrow L_{\mathbb{R}}$. The discriminant group of $L$ is defined to be $L^{\vee} / L$. A lattice $L$ is unimodular if $L^{\vee} / L$ is trivial. A lattice $L$ of rank $n$ is hyperbolic if $n>1$ and the signature of $L_{\mathbb{R}}$ is $(1, n-1)$, whereas $L$ is negative-definite if the signature is $(0, n)$.

A lattice $L$ is even if $\langle x, x\rangle \in 2 \mathbb{Z}$ for all $x \in L$. Suppose that $L$ is even. Then the discriminant form $q_{L}: L^{\vee} / L \rightarrow \mathbb{Q} / 2 \mathbb{Z}$ of $L$ is defined by $q_{L}(x \bmod L):=\langle x, x\rangle \bmod$ $2 \mathbb{Z}$ for $x \in L^{\vee}$. See [22] for the basic properties of discriminant forms. We denote by $\mathrm{O}\left(q_{L}\right)$ the automorphism group of the finite quadratic form $q_{L}$. We regard $L^{\vee}$ as a submodule of $L_{\mathbb{Q}}$, and let $\mathrm{O}(L)$ act on $L^{\vee}$ from the right. We have a natural homomorphism $\eta_{L}: \mathrm{O}(L) \rightarrow \mathrm{O}\left(q_{L}\right)$.

A vector $r \in L$ with $\langle r, r\rangle=-2$ is called a root. A root $r \in L$ defines a reflection

$$
s_{r}: x \mapsto x+\langle x, r\rangle r
$$


that belongs to $\mathrm{O}(L)$. The Weyl group $W(L)$ of $L$ is defined to be the subgroup of $\mathrm{O}(L)$ generated by the reflections $s_{r}$ with respect to all the roots $r$ of $L$.

Let $L$ be an even hyperbolic lattice. A positive cone of $L$ is one of the two connected components of $\left\{x \in L_{\mathbb{R}} \mid\langle x, x\rangle>0\right\}$. We fix a positive cone $\mathcal{P}$ of $L$. Let $\mathrm{O}^{+}(L)$ denote the stabilizer subgroup of $\mathcal{P}$ in $\mathrm{O}(L)$. Then $W(L)$ acts on $\mathcal{P}$. For a root $r \in L$, we put $(r)^{\perp}:=\{x \in \mathcal{P} \mid\langle x, r\rangle=0\}$. The following is obvious.

Proposition 2.1 The family $\left\{(r)^{\perp} \mid r\right.$ is a root of $\left.L\right\}$ of hyperplanes of $\mathcal{P}$ is locally finite in $\mathcal{P}$.

A standard fundamental domain of the action of $W(L)$ on $\mathcal{P}$ is the closure in $\mathcal{P}$ of a connected component of $\mathcal{P} \backslash \cup_{r}(r)^{\perp}$, where $r$ runs through the set of all roots. Let $D$ be one of the standard fundamental domains of $W(L)$. We put

$$
\operatorname{aut}(D):=\left\{g \in \mathrm{O}^{+}(L) \mid D^{g}=D\right\} .
$$

Then we have $\mathrm{O}^{+}(L)=W(L) \rtimes \operatorname{aut}(D)$.

\section{$2.2 \quad V_{\mathbb{R}}$-chambers}

Let $V$ be a $\mathbb{Q}$-vector space of dimension $n>0$, and let $V^{*}$ denote the dual $\mathbb{Q}$-vector space $\operatorname{Hom}(V, \mathbb{Q})$. We put $V_{\mathbb{R}}:=V \otimes \mathbb{R}$. For a non-zero linear form $f \in V^{*} \backslash\{0\}$, we put

$$
H_{f}:=\left\{x \in V_{\mathbb{R}} \mid f(x) \geq 0\right\}, \quad[f]^{\perp}:=\left\{x \in V_{\mathbb{R}} \mid f(x)=0\right\}=\partial H_{f} .
$$

Definition 2.2 A closed subset $\bar{C}$ of $V_{\mathbb{R}}$ is called a $V_{\mathbb{R}}$-chamber if $\bar{C}$ contains a nonempty open subset of $V_{\mathbb{R}}$, and there exists a subset $\mathcal{F}$ of $V^{*} \backslash\{0\}$ such that

$$
\bar{C}=\bigcap_{f \in \mathcal{F}} H_{f} .
$$

When this is the case, we say that $\mathcal{F}$ defines the $V_{\mathbb{R}}$-chamber $\bar{C}$.

Suppose that a subset $\mathcal{F}$ of $V^{*} \backslash\{0\}$ defines a $V_{\mathbb{R}}$-chamber $\bar{C}$. We assume that

$$
H_{f} \neq H_{f^{\prime}} \text { for distinct } f, f^{\prime} \in \mathcal{F} \text {. }
$$

We say that an element $f$ of $V^{*} \backslash\{0\}$ defines a wall of $\bar{C}$ if $\bar{C}$ is contained in $H_{f}$ and $\bar{C} \cap[f]^{\perp}$ contains a non-empty open subset of $[f]^{\perp}$. When this is the case, we call $\bar{C} \cap[f]^{\perp}$ the wall of $\bar{C}$ defined by $f$. By the assumption (2.1), we see that $f_{0} \in \mathcal{F}$ defines a wall of $\bar{C}$ if and only if there exists a point $x \in V$ such that $f_{0}(x)<0$ and $f(x) \geq 0$ for all $f \in \mathcal{F} \backslash\left\{f_{0}\right\}$. Hence we have the following.

Algorithm 2.3 Suppose that a $V_{\mathbb{R}}$-chamber $\bar{C}$ is defined by a finite subset $\mathcal{F}$ of $V^{*} \backslash\{0\}$ satisfying (2.1). Then an element $f_{0} \in \mathcal{F}$ defines $a$ wall of $C$ if and only if the solution of the following linear programming problem on $V$ over $\mathbb{Q}$ is unbounded to $-\infty$ :

Find the minimal value of $f_{0}(x)$

subject to the constraints $f(x) \geq 0$ for all $f \in \mathcal{F} \backslash\left\{f_{0}\right\}$. 
Let $\bar{C}$ and $\mathcal{F}$ be as above. We define the faces of dimension $k$ of $\bar{C}$ for $k=n-1, \ldots, 1$ by descending induction on $k$. The following is obvious.

Lemma 2.4 Suppose that $n>1$, and that $f_{0} \in \mathcal{F}$ defines a wall of $\bar{C}$. For $g \in V^{*}$, let $\left.g\right|_{f_{0}^{\perp}}: f_{0}^{\perp} \rightarrow \mathbb{Q}$ denote the restriction of $g$ to the hyperplane $f_{0}^{\perp}$ of $V$. Then the wall $\bar{C} \cap\left[f_{0}\right]^{\perp}$ of $\bar{C}$ defined by $f_{0}$ is an $\left[f_{0}\right]^{\perp}$-chamber defined by

$$
\left.\mathcal{F}\right|_{f_{0}^{\perp}}:=\left\{\left.g\right|_{f_{0}^{\perp}}|g \in \mathcal{F}, g|_{f_{0}^{\perp}} \neq 0\right\} \text {. }
$$

The faces of $\bar{C}$ of dimension $n-1$ are defined to be the walls of $\bar{C}$. Suppose that $0<k<n-1$, and let $F$ be a $(k+1)$-dimensional face of $\bar{C}$. Let $\langle F\rangle$ denote the minimal linear subspace of $V$ containing $F$. We assume that (1) the linear space $\langle F\rangle$ is of dimension $k+1$, (2) $F$ is equal to the closed subset $\bar{C} \cap\langle F\rangle$ of $\bar{C}$, and (3) $F$ is an $(\langle F\rangle \otimes \mathbb{R})$-chamber defined by the subset $\left.\mathcal{F}\right|_{\langle F\rangle}:=\left\{\left.g\right|_{\langle F\rangle}|g \in \mathcal{F}, g|_{\langle F\rangle} \neq 0\right\}$ of $\operatorname{Hom}(\langle F\rangle, \mathbb{Q}) \backslash\{0\}$, where $\left.g\right|_{\langle F\rangle}$ is the restriction of $g$ to $\langle F\rangle$. Then the walls of the $(\langle F\rangle \otimes \mathbb{R})$-chamber $F$ are defined. A face of dimension $k$ of $\bar{C}$ is defined to be a wall of a $(k+1)$-dimensional face of $\bar{C}$. It is obvious that $k$-dimensional faces satisfy the assumptions (1)-(3), and hence the induction proceeds.

When $\mathcal{F}$ is finite, we can calculate all the faces of $\bar{C}$ by using Algorithm 2.3 iteratively.

Remark 2.5 At every step of iteration, we must remove redundant elements from $\left.\mathcal{F}\right|_{\langle F\rangle}$ to obtain a subset $\left.\mathcal{F}_{\langle F\rangle}^{\prime} \subset \mathcal{F}\right|_{\langle F\rangle}$ that defines the walls of $F$ and satisfies (2.1).

\subsection{Chambers}

Let $V$ be as in the previous subsection. Suppose that $n>1$, and that $V$ is equipped with a non-degenerate symmetric bilinear form $\langle\cdot, \cdot\rangle: V \times V \rightarrow \mathbb{Q}$ such that $V_{\mathbb{R}}=V \otimes \mathbb{R}$ is of signature $(1, n-1)$. By $\langle\cdot, \cdot\rangle$, we identify $V$ and $V^{*}$. In particular, for a non-zero vector $v$ of $V$, we put

$$
H_{v}:=\left\{x \in V_{\mathbb{R}} \mid\langle v, x\rangle \geq 0\right\}, \quad[v]^{\perp}:=\left\{x \in V_{\mathbb{R}} \mid\langle v, x\rangle=0\right\}=\partial H_{v} .
$$

Let $\mathcal{P}_{V}$ be one of the two connected components of $\left\{x \in V_{\mathbb{R}} \mid\langle x, x\rangle>0\right\}$, and let $\overline{\mathcal{P}}_{V}$ denote the closure of $\mathcal{P}_{V}$ in $V_{\mathbb{R}}$. For a non-zero vector $v$ of $V$, we put $(v)^{\perp}:=[v]^{\perp} \cap \mathcal{P}_{V}$, which is non-empty if and only if $\langle v, v\rangle<0$.

Definition 2.6 A closed subset $C$ of $\mathcal{P}_{V}$ is said to be a chamber if there exists a subset $\mathcal{F}$ of $V \backslash\{0\}$ with the following properties.

(i) The family $\left\{(v)^{\perp} \mid v \in \mathcal{F},\langle v, v\rangle<0\right\}$ of hyperplanes of the positive cone $\mathcal{P}_{V}$ is locally finite in $\mathcal{P}_{V}$.

(ii) Under the identification $V=V^{*}$, the set $\mathcal{F}$ defines a $V_{\mathbb{R}}$-chamber $\bar{C}$ such that

$$
\bar{C} \subset \overline{\mathcal{P}}_{V} \text { and } C=\mathcal{P}_{V} \cap \bar{C} \text {. }
$$

When this is the case, we say that the chamber $C$ is defined by $\mathcal{F}$.

Remark 2.7 A $V_{\mathbb{R}}$-chamber $\bar{C}$ satisfies $\bar{C} \subset \overline{\mathcal{P}}_{V}$ if and only if $\bar{C} \cap \mathcal{P}_{V} \neq \varnothing$ and $\bar{C} \cap \partial \overline{\mathcal{P}}_{V}$ is contained in the union of one-dimensional faces of $\bar{C}$. 
Let $C$ be a chamber defined by $\mathcal{F} \subset V \backslash\{0\}$. Let $F$ be a $k$-dimensional face of $\bar{C}$. If $C \cap F \neq \varnothing$, we say that $C \cap F$ is a face of $C$ of dimension $k$. Note that, by (2.2), if $k>1$, then $C \cap F$ is a face of $C$. In particular, since $n>1$, if $\bar{C} \cap[u]^{\perp}$ is a wall of $\bar{C}$ defined by $u \in V \backslash\{0\}$, then $C \cap(u)^{\perp}$ is called the wall of $C$ defined by $u$.

When $k=1$, we may have $C \cap F=\varnothing$.

Definition 2.8 A one-dimensional face $F$ of $\bar{C}$ contained in $\bar{C} \backslash C=\bar{C} \cap \partial \overline{\mathcal{P}}_{V}$ is called an ideal face of $C$. By abuse of language, an ideal face of $C$ is also regarded as a face of $C$.

\subsection{Chambers of a Hyperbolic Lattice}

Let $L$ be an even hyperbolic lattice with a positive cone $\mathcal{P}$. Applying the above definition to $V=L_{\mathbb{Q}}$, we have the notion of chambers and their faces. By a chamber of $L$ we mean a chamber of $L_{\mathbb{Q}}$.

Definition 2.9 We define the automorphism group of a chamber $C$ of $L$ by

$$
\operatorname{aut}(C):=\left\{g \in \mathrm{O}^{+}(L) \mid C^{g}=C\right\}
$$

We put $L_{\text {prim }}^{\vee}:=\left\{v \in L^{\vee} \mid v\right.$ is primitive in $\left.L^{\vee}\right\}$. Then we have a canonical projection $L_{\mathbb{Q}} \backslash\{0\} \rightarrow L_{\text {prim }}^{\vee}, v \mapsto \widetilde{v}$ such that $H_{v}=H_{\widetilde{v}}$ holds for all $v \in L_{\mathbb{Q}} \backslash\{0\}$.

Definition 2.10 Let $C \cap(u)^{\perp}$ be a wall of a chamber $C$ of $L$ defined by $u \in L_{\mathbb{Q}}$. A vector $v \in L^{\vee}$ is called the primitive defining vector of the wall $C \cap(u)^{\perp}$ if $v$ is the vector of $L_{\text {prim }}^{\vee}$ satisfying $H_{v}=H_{u}$. By definition, each wall $C \cap(u)^{\perp}$ of $C$ has a unique primitive defining vector $\widetilde{u}$.

If $\mathcal{F} \subset L_{\mathbb{Q}} \backslash\{0\}$ defines a chamber $C$, then so does the set $\widetilde{\mathcal{F}}:=\{\widetilde{v} \mid v \in \mathcal{F}\}$. Assumption (2.1) holds automatically for $\widetilde{\mathcal{F}}$. Hence converting $\mathcal{F}$ to $\widetilde{\mathcal{F}}$ is a convenient method to achieve the property (2.1) when we use Algorithm 2.3 iteratively to determine the faces of a chamber (see Remark 2.5).

\section{Even Unimodular Hyperbolic Lattices $L_{10}$ and $L_{26}$}

For each positive integer $n$ with $n \equiv 2 \bmod 8$, let $L_{n}$ denote an even unimodular hyperbolic lattice of rank $n$, which is unique up to isomorphism. The lattice $L_{2}$ is denoted by $U$. We fix a basis $f_{1}, f_{2}$ of $U$ such that the Gram matrix of $U$ with respect to $f_{1}, f_{2}$ is $\left[\begin{array}{ll}0 & 1 \\ 1 & 0\end{array}\right]$.

\subsection{The Lattice $L_{10}$}

Let $E_{8}$ denote the negative-definite even unimodular lattice of rank 8 with the standard basis $e_{1}, \ldots, e_{8}$, whose intersection numbers are given by the Dynkin diagram in Figure 3.1. We use $f_{1}, f_{2}, e_{1}, \ldots, e_{8}$ as a basis of $L_{10}:=U \oplus E_{8}$, and put 


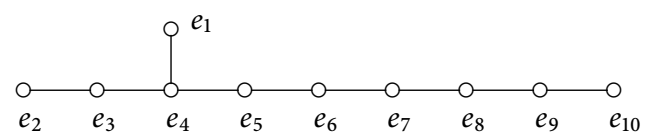

Figure 3.1: Walls of the Vinberg chamber $D_{10}$

$$
\begin{aligned}
e_{9} & :=[1,0,-3,-2,-4,-6,-5,-4,-3,-2] \\
e_{10} & :=[-1,1,0,0,0,0,0,0,0,0] \\
w_{10} & :=[31,30,-68,-46,-91,-135,-110,-84,-57,-29] .
\end{aligned}
$$

We have $\left\langle e_{9}, e_{9}\right\rangle=\left\langle e_{10}, e_{10}\right\rangle=-2,\left\langle w_{10}, w_{10}\right\rangle=1240$, and

$$
\mathcal{W}_{10}:=\left\{r \in L_{10} \mid\left\langle r, w_{10}\right\rangle=1,\langle r, r\rangle=-2\right\}=\left\{e_{1}, \ldots, e_{10}\right\} .
$$

The roots $e_{1}, \ldots, e_{10}$ form the Dynkin diagram in Figure 3.1. Let $\mathcal{P}_{10}$ be the positive cone of $L_{10}$ containing $w_{10}$, and $\overline{\mathcal{P}}_{10}$ the closure of $\mathcal{P}_{10}$ in $L_{10} \otimes \mathbb{R}$. We put

$$
\bar{D}_{10}:=\left\{x \in L_{10} \otimes \mathbb{R} \mid\left\langle x, e_{i}\right\rangle \geq 0 \text { for } i=1, \ldots, 10\right\}, \quad D_{10}:=\bar{D}_{10} \cap \mathcal{P}_{10} .
$$

Theorem 3.1 (Vinberg [32]) The closed subset $D_{10}$ of $\mathcal{P}_{10}$ is a chamber. The chamber $D_{10}$ is a standard fundamental domain of the action of $W\left(L_{10}\right)$ on $\mathcal{P}_{10}$. Each vector $e_{i}$ of $\mathcal{W}_{10}$ defines a wall of $D_{10}$.

Since the diagram of the walls of $D_{10}$ in Figure 3.1 has no symmetries, the automorphism group aut $\left(D_{10}\right)$ of $D_{10}$ is trivial. Hence $\mathrm{O}^{+}\left(L_{10}\right)$ is equal to $W\left(L_{10}\right)$, which is generated by the reflections with respect to the roots $e_{1}, \ldots, e_{10}$.

Definition 3.2 A standard fundamental domain of the action of $W\left(L_{10}\right)$ on $\mathcal{P}_{10}$ is called a Vinberg chamber.

\subsection{The Lattice $L_{26}$}

Let $\Lambda$ be the negative-definite Leech lattice; that is, the unique even negative-definite unimodular lattice of rank 24 with no roots. As a basis of $\Lambda$, we choose the row vectors $\lambda_{1}, \ldots, \lambda_{24}$ of the matrix given in Table 3.1 and consider them as elements of the quadratic space $\mathbb{R}^{24}$ with the negative-definite inner product

$$
(x, y) \mapsto-\left(x_{1} y_{1}+\cdots+x_{24} y_{24}\right) / 8
$$

This basis is constructed from the extended binary Golay code in the space of $\mathbb{F}_{2}$-valued functions on $\mathbb{P}^{1}\left(\mathbb{F}_{23}\right)=\{\infty\} \cup \mathbb{F}_{23}$, where the value at $\infty$ is at the first coordinate of each row vector $[9, \S 2.8]$. We put $L_{26}:=U \oplus \Lambda$. Then the vectors $f_{1}, f_{2}, \lambda_{1}, \ldots, \lambda_{24}$ form a basis of $L_{26}$, which we will use throughout this paper. We put

$$
w_{26}:=f_{1}, \quad \mathcal{W}_{26}:=\left\{r \in L_{26} \mid\left\langle r, w_{26}\right\rangle=1,\langle r, r\rangle=-2\right\} .
$$




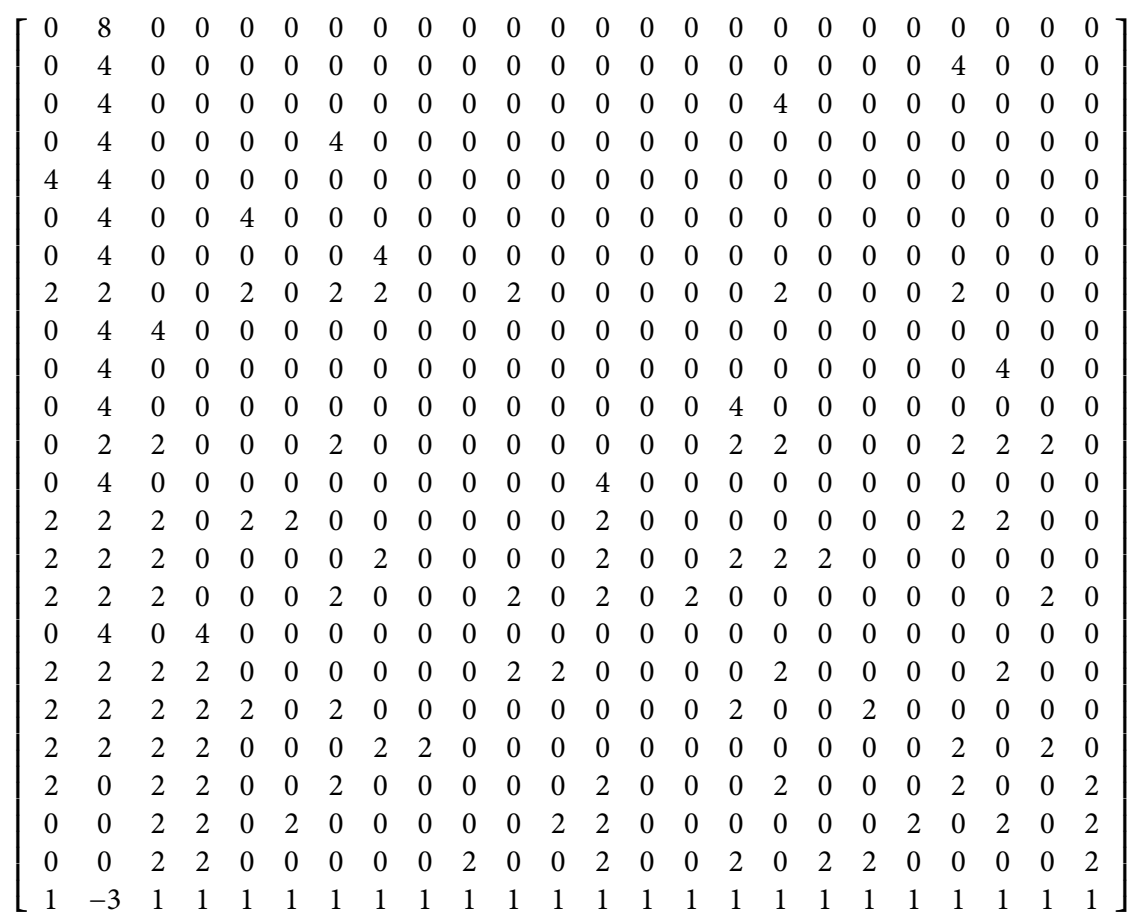

Table 3.1: Basis of the Leech lattice $\Lambda$.

Note that $\left\langle w_{26}, w_{26}\right\rangle=0$. Let $\mathcal{P}_{26}$ be the positive cone of $L_{26}$ that contains $w_{26}$ in its closure $\overline{\mathcal{P}}_{26}$ in $L_{26} \otimes \mathbb{R}$. We then put

$$
\bar{D}_{26}:=\left\{x \in L_{26} \otimes \mathbb{R} \mid\langle x, r\rangle \geq 0 \text { for all } r \in \mathcal{W}_{26}\right\}, \quad D_{26}:=\bar{D}_{26} \cap \mathcal{P}_{26} .
$$

Theorem 3.3 (Conway [5]) The closed subset $D_{26}$ of $\mathcal{P}_{26}$ is a chamber. The chamber $D_{26}$ is a standard fundamental domain of the action of $W\left(L_{26}\right)$ on $\mathcal{P}_{26}$. Each vector $r_{\lambda}$ of $\mathcal{W}_{26}$ defines a wall of $D_{26}$.

Definition 3.4 A standard fundamental domain of the action of $W\left(L_{26}\right)$ on $\mathcal{P}_{26}$ is called a Conway chamber.

A chamber $D^{\prime}$ in $\mathcal{P}_{26}$ is a Conway chamber if and only if $D^{\prime}$ is equal to $D_{26}^{g}$ for some $g \in W\left(L_{26}\right)$. The Weyl vector $w^{\prime}$ of a Conway chamber $D^{\prime}=D_{26}^{g}$ is defined to be $w_{26}^{g}$, which is characterized by the following property:

$$
D^{\prime}=\left\{x \in \mathcal{P}_{26} \mid\langle x, r\rangle \geq 0 \text { for all roots } r \in L_{26} \text { satisfying }\left\langle w^{\prime}, r\right\rangle=1\right\} .
$$


On an Enriques Surface Associated With a Quartic Hessian Surface

\section{Borcherds's Method for K3 Surfaces}

\subsection{Borcherds's Method}

We review the method due to Borcherds $[3,4]$ to calculate a standard fundamental domain of the Weyl group of an even hyperbolic lattice. See also [27] for the algorithmic description of Borcherds's method.

Definition 4.1 In the following, a tessellation of a subset $N$ of a positive cone $\mathcal{P}$ of a hyperbolic lattice means a decomposition of $N$ into a union of chambers such that, if $D$ and $D^{\prime}$ are distinct chambers in the decomposition, then the interiors of $D$ and $D^{\prime}$ are disjoint.

Let $S$ be an even hyperbolic lattice. Suppose that we have a primitive embedding $i: S \hookrightarrow L_{26}$ of $S$ into the even unimodular hyperbolic lattice $L_{26}$ of rank 26 . To simplify notation, we use the same letter $i$ to denote $i \otimes \mathbb{R}: S \otimes \mathbb{R} \hookrightarrow L_{26} \otimes \mathbb{R}$. We put

$$
\mathcal{P}_{S}:=i^{-1}\left(\mathcal{P}_{26}\right),
$$

where $\mathcal{P}_{26}$ is the fixed positive cone of $L_{26}$. Then $\mathcal{P}_{S}$ is a positive cone of $S$. We denote by $\operatorname{pr}_{S}: L_{26} \otimes \mathbb{R} \rightarrow S \otimes \mathbb{R}$ the orthogonal projection to $S \otimes \mathbb{R}$. Note that $\operatorname{pr}_{S}\left(L_{26}\right) \subset S^{\vee}$. Note also that, for $v \in L_{26} \otimes \mathbb{R}$, we have

$$
i^{-1}\left((v)^{\perp}\right)=\left(\operatorname{pr}_{S}(r)\right)^{\perp}=\left\{x \in \mathcal{P}_{S} \mid\left\langle x, \operatorname{pr}_{S}(v)\right\rangle=0\right\},
$$

and hence $i^{-1}\left((v)^{\perp}\right) \neq \varnothing$ holds if and only if $\left\langle\operatorname{pr}_{S}(v), \operatorname{pr}_{S}(v)\right\rangle<0$. We put

$$
\mathcal{R}_{S}:=\left\{\operatorname{pr}_{S}(r) \mid r \text { is a root of } L_{26} \text { such that }\left\langle\operatorname{pr}_{S}(r), \operatorname{pr}_{S}(r)\right\rangle<0\right\} \text {. }
$$

Then the family of hyperplanes $\mathcal{H}_{S}:=\left\{\left(\operatorname{pr}_{S}(r)\right)^{\perp} \mid \operatorname{pr}_{S}(r) \in \mathcal{R}_{S}\right\}$ is locally finite in $\mathcal{P}_{S}$ by Proposition 2.1. We investigate the tessellation of $\mathcal{P}_{S}$ obtained by this family of hyperplanes.

A Conway chamber $D^{\prime}=D_{26}^{g}$ in $\mathcal{P}_{26}$ is said to be non-degenerate with respect to $S$ if the closed subset $i^{-1}\left(D^{\prime}\right)$ of $\mathcal{P}_{S}$ contains a non-empty open subset of $\mathcal{P}_{S}$. When this is the case, the closed subset $i^{-1}\left(D^{\prime}\right)$ of $\mathcal{P}_{S}$ is a chamber in $\mathcal{P}_{S}$ defined by the following subset of $\mathcal{R}_{S}$, where $w^{\prime}$ is the Weyl vector of $D^{\prime}$ :

$$
\left\{\operatorname{pr}_{S}(r) \mid r \text { is a root of } L_{26} \text { satisfying }\left\langle r, w^{\prime}\right\rangle=1 \text { and }\left\langle\operatorname{pr}_{S}(r), \operatorname{pr}_{S}(r)\right\rangle<0\right\} \text {. }
$$

Definition 4.2 A chamber of $\mathcal{P}_{S}$ is said to be an induced chamber if it is of the form $i^{-1}\left(D^{\prime}\right)$, where $D^{\prime}$ is a Conway chamber, non-degenerate with respect to $S$.

Since $\mathcal{P}_{26}$ is tessellated by Conway chambers, the cone $\mathcal{P}_{S}$ is tessellated by induced chambers. Each induced chamber is the closure in $\mathcal{P}_{S}$ of a connected component of $\mathcal{P}_{S} \backslash \bigcup_{(v)^{\perp} \in \mathcal{H}_{S}}(v)^{\perp}$. The following is the main result of [27].

Proposition 4.3 Suppose that the orthogonal complement of $S$ in $L_{26}$ cannot be embedded into the negative-definite Leech lattice $\Lambda$. Then each induced chamber $i^{-1}\left(D^{\prime}\right)$ has only a finite number of walls, and the set of walls can be calculated from the Weyl vector $w^{\prime}$ of $D^{\prime}$. 
Every root $r$ of $S$ satisfies $r=\operatorname{pr}_{S}(r)$ and hence belongs to $\mathcal{R}_{S}$. Therefore we have the following.

Proposition 4.4 Each standard fundamental domain of the action of $W(S)$ on $\mathcal{P}_{S}$ is tessellated by induced chambers.

For each wall of an induced chamber $i^{-1}\left(D^{\prime}\right)$, there exists a unique induced chamber that shares the wall with $i^{-1}\left(D^{\prime}\right)$. More precisely, if $v \in \mathcal{R}_{S}$ defines a wall

$$
i^{-1}\left(D^{\prime}\right) \cap(v)^{\perp}
$$

of $i^{-1}\left(D^{\prime}\right)$, then there exists a unique induced chamber $i^{-1}\left(D^{\prime \prime}\right)$ such that $-v \in \mathcal{R}_{S}$ defines a wall $i^{-1}\left(D^{\prime \prime}\right) \cap(-v)^{\perp}$ of $i^{-1}\left(D^{\prime \prime}\right)$, and that we have

$$
i^{-1}\left(D^{\prime}\right) \cap(v)^{\perp}=i^{-1}\left(D^{\prime \prime}\right) \cap(-v)^{\perp} .
$$

Definition 4.5 We call the chamber $i^{-1}\left(D^{\prime \prime}\right)$ satisfying the properties above the induced chamber adjacent to $i^{-1}\left(D^{\prime}\right)$ across the wall $i^{-1}\left(D^{\prime}\right) \cap(v)^{\perp}$.

Definition 4.6 We say that the primitive embedding $i: S \hookrightarrow L_{26}$ is of simple Borcherds type if, for any two induced chambers $i^{-1}\left(D^{\prime}\right)$ and $i^{-1}\left(D^{\prime \prime}\right)$, there exists an isometry $g \in \mathrm{O}^{+}(S)$ such that $i^{-1}\left(D^{\prime \prime}\right)^{g}=i^{-1}\left(D^{\prime}\right)$.

In order to prove that $i: S \hookrightarrow L_{26}$ is of simple Borcherds type, it is enough to choose an induced chamber $i^{-1}\left(D^{\prime}\right)$ and show that, for each wall $i^{-1}\left(D^{\prime}\right) \cap(v)^{\perp}$ of $i^{-1}\left(D^{\prime}\right)$, there exists an element $g \in \mathrm{O}^{+}(S)$ extending to an isometry of $L_{26}$ such that $i^{-1}\left(D^{\prime}\right)^{g}$ is the induced chamber adjacent to $i^{-1}\left(D^{\prime}\right)$ across the wall $i^{-1}\left(D^{\prime}\right) \cap(v)^{\perp}$.

\subsection{Torelli's Theorem for K3 Surfaces}

We recall how to apply Torelli's theorem [25] for complex $K 3$ surfaces to the study of the automorphism groups. Let $X$ be a complex algebraic $K 3$ surface. We denote by $S_{X}$ the Néron-Severi lattice of $X$. Suppose that rank $S_{X}>1$. Then $S_{X}$ is an even hyperbolic lattice. For a divisor $D$ on $X$, we denote $[D] \in S_{X}$ the class of $D$. Let $\mathcal{P}_{X}$ be the positive cone of $S_{X}$ containing an ample class. We put

$$
N(X):=\left\{x \in \mathcal{P}_{X} \mid\langle x,[C]\rangle \geq 0 \text { for all curves } C \text { on } X\right\} .
$$

It is well known that $N(X)$ is a chamber of $S_{X}$, that $N(X)$ is a standard fundamental domain of the action of $W\left(S_{X}\right)$ on $\mathcal{P}_{X}$, and that the correspondence $C \mapsto N(X) \cap$ $([C])^{\perp}$ gives a bijection from the set of smooth rational curves $C$ on $X$ to the set of walls of the chamber $N(X)$.

Let $\operatorname{Aut}(X)$ denote the automorphism group of $X$ acting on $X$ from the left. Let $\operatorname{aut}(X)$ be the image of the natural representation $\varphi_{X}: \operatorname{Aut}(X) \rightarrow \mathrm{O}^{+}\left(S_{X}\right)$ defined by the pullback of the classes of divisors. Then we have aut $(X) \subset \operatorname{aut}(N(X))$, where aut $(N(X))$ is the automorphism group of the chamber $N(X)$.

Let $T_{X}$ denote the orthogonal complement of $S_{X}=H^{2}(X, \mathbb{Z}) \cap H^{1,1}(X)$ in the even unimodular lattice $H^{2}(X, \mathbb{Z})$ with the cup product, and let $\omega_{X}$ be a generator of the one-dimensional subspace $H^{2,0}(X)$ of $T_{X} \otimes \mathbb{C}$. We put

$$
\mathrm{O}^{\omega}\left(T_{X}\right):=\left\{g \in \mathrm{O}\left(T_{X}\right) \mid \omega_{X}^{g} \in \mathbb{C} \omega_{X}\right\} .
$$


By [22], there exists a unique isomorphism $\sigma_{X}: q_{S_{X}} \stackrel{\sim}{\rightarrow}-q_{T_{X}}$ of finite quadratic forms such that the graph of $\sigma_{X}$ is the image of $H^{2}(X, \mathbb{Z}) \subset S_{X}^{\vee} \oplus T_{X}^{\vee}$ by the natural projection to $\left(S_{X}^{\vee} / S_{X}\right) \oplus\left(T_{X}^{\vee} / T_{X}\right)$. We denote by $\sigma_{X *}: \mathrm{O}\left(q_{S_{X}}\right) \stackrel{\sim}{\rightarrow} \mathrm{O}\left(q_{T_{X}}\right)$ the isomorphism induced by $\sigma_{X}$. Recall that $\eta_{L}: \mathrm{O}(L) \rightarrow \mathrm{O}\left(q_{L}\right)$ denotes the natural homomorphism. By [22] again, an element $g$ of $\mathrm{O}\left(S_{X}\right)$ extends to an isometry of $H^{2}(X, \mathbb{Z})$ preserving the Hodge structure if and only if

$$
\sigma_{X *}\left(\eta_{S_{X}}(g)\right) \in \eta_{T_{X}}\left(\mathrm{O}^{\omega}\left(T_{X}\right)\right) .
$$

By the Torelli theorem for complex $K 3$ surfaces [25], we have the following.

Theorem 4.7 (i) An element $g$ of $\operatorname{aut}(N(X))$ belongs to aut $(X)$ if and only if $g$ satisfies condition (4.1).

(ii) The kernel of $\varphi_{X}: \operatorname{Aut}(X) \rightarrow \operatorname{aut}(X)$ is isomorphic to the group

$$
\left\{g \in \mathrm{O}^{\omega}\left(T_{X}\right) \mid \eta_{T_{X}}(g)=1\right\} \text {. }
$$

Theorem 4.7 enables us to calculate $\operatorname{Aut}(X)$ from aut $(N(X))$. Kondo [15] studied the automorphism group of a generic Jacobian Kummer surface $X$ by finding a primitive embedding $S_{X} \rightarrow L_{26}$ of simple Borcherds type and calculating aut $(N(X))$. Since then, many authors have studied the automorphism groups of $K 3$ surfaces by this method. See [27] and the references therein. On the other hand, in [11, 27], this method was generalized to primitive embeddings $S_{X} \rightarrow L_{26}$ that are not of simple Borcherds type.

\section{Computational Study of Geometry of an Enriques Surface}

\subsection{Borcherds's Method for an Enriques Surface}

Suppose that a $K 3$ surface $X$ has an Enriques involution $\varepsilon \in \operatorname{Aut}(X)$, and let $g_{\varepsilon}:=$ $\varphi(\varepsilon) \in \operatorname{aut}(X)$ denote its action on $S_{X}$. Let $Y:=X /\langle\varepsilon\rangle$ be the quotient of $X$ by $\varepsilon$, and let $\pi: X \rightarrow Y$ denote the quotient morphism. Let $S_{Y}$ denote the lattice of numerical equivalence classes of divisors on $Y$. It is well known that $S_{Y}$ is isomorphic to the even unimodular hyperbolic lattice $L_{10}$ of rank 10. Let $\mathcal{P}_{Y}$ be the positive cone of $S_{Y}$ containing an ample class. We put

$$
N(Y):=\left\{y \in \mathcal{P}_{Y} \mid\langle y,[C]\rangle \geq 0 \text { for all curves } C \text { on } Y\right\} .
$$

Let aut $(Y)$ denote the image of the natural representation $\varphi_{Y}: \operatorname{Aut}(Y) \rightarrow \mathrm{O}^{+}\left(S_{Y}\right)$ defined by the pullback of the classes of divisors. We have aut $(Y) \subset \operatorname{aut}(N(Y))$. We consider the following two primitive sublattices of $S_{X}$ :

$$
S_{X}^{+}:=\left\{v \in S_{X} \mid v^{g_{\varepsilon}}=v\right\}, \quad S_{X}^{-}:=\left\{v \in S_{X} \mid v^{g_{\varepsilon}}=-v\right\} .
$$

The homomorphism $\pi^{*}: S_{Y} \rightarrow S_{X}$ induces an isomorphism of lattices

$$
\pi^{*}: S_{Y}(2) \stackrel{\sim}{\longrightarrow} S_{X}^{+},
$$

by which we regard $S_{Y}$ as a $\mathbb{Z}$-submodule of $S_{X}$. In particular, we have

$$
\mathcal{P}_{Y}=\left(S_{Y} \otimes \mathbb{R}\right) \cap \mathcal{P}_{X}, \quad N(Y)=\mathcal{P}_{Y} \cap N(X) .
$$


(The second quality follows from the projection formula and the fact that $\pi$ is finite.) We use $\langle\cdot, \cdot\rangle_{X}$ and $\langle\cdot, \cdot\rangle_{Y}$ to denote the intersection forms of $S_{X}$ and $S_{Y}$, respectively, so that, for $y, y^{\prime} \in S_{Y} \otimes \mathbb{R}$, we have $\left\langle y, y^{\prime}\right\rangle_{X}=2\left\langle y, y^{\prime}\right\rangle_{Y}$.

For a group $G$ and an element $g \in G$, we denote by $Z_{G}(g)$ the centralizer of $g$ in $G$. We have a natural isomorphism $\operatorname{Aut}(Y) \cong Z_{\text {Aut }(X)}(\varepsilon) /\langle\varepsilon\rangle$. Hence we have the following.

Proposition 5.1 Suppose that the representation $\varphi_{X}: \operatorname{Aut}(X) \rightarrow \operatorname{aut}(X)$ is an isomorphism. Then we have a surjective homomorphism $\zeta: Z_{\text {aut }(X)}\left(g_{\varepsilon}\right) /\left\langle g_{\varepsilon}\right\rangle \rightarrow \operatorname{aut}(Y)$ defined by the commutative diagram

$$
\begin{array}{ccc}
Z_{\operatorname{Aut}(X)}(\varepsilon) /\langle\varepsilon\rangle & \cong & Z_{\text {aut }(X)}\left(g_{\varepsilon}\right) /\left\langle g_{\varepsilon}\right\rangle \\
2 \downarrow & & \downarrow \zeta \\
\operatorname{Aut}(Y) & \longrightarrow & \operatorname{aut}(Y) .
\end{array}
$$

The homomorphism $\zeta$ is defined as follows. If $g \in Z_{\text {aut }(X)}\left(g_{\varepsilon}\right)$, then $S_{Y}^{g}=S_{Y}$ holds, and the restriction $\left.g\right|_{S_{Y}} \in \mathrm{O}\left(S_{Y}\right)$ of $g$ to $S_{Y}$ gives $\zeta(g) \in \operatorname{aut}(Y)$.

We denote the orthogonal projection to $S_{Y} \otimes \mathbb{R}=S_{X}^{+} \otimes \mathbb{R}$ by

$$
\mathrm{pr}^{+}: S_{X} \otimes \mathbb{R} \longrightarrow S_{Y} \otimes \mathbb{R} \text {. }
$$

Suppose that we have a primitive embedding $i: S_{X} \hookrightarrow L_{26}$, and hence $\mathcal{P}_{X}$ is tessellated by induced chambers. The composite of the primitive embeddings $\pi^{*}: S_{Y}(2) \hookrightarrow S_{X}$ and $i: S_{X} \hookrightarrow L_{26}$ gives a primitive embedding of $S_{Y}(2)$ into $L_{26}$. By this embedding, the notion of induced chambers in $\mathcal{P}_{Y}$ is defined. Recall that $\mathrm{pr}_{S}: L_{26} \otimes \mathbb{R} \rightarrow S_{X} \otimes \mathbb{R}$ is the orthogonal projection. Let $\mathrm{pr}_{S}^{+}: L_{26} \otimes \mathbb{R} \rightarrow S_{Y} \otimes \mathbb{R}$ denote the composite of $\mathrm{pr}_{S}$ and $\mathrm{pr}^{+}$. Then the tessellation of $\mathcal{P}_{Y}$ by induced chambers is given by the locally finite family of hyperplanes

$$
\left\{\left(\operatorname{pr}_{S}^{+}(r)\right)^{\perp} \mid r \text { is a root of } L_{26} \text { such that }\left\langle\operatorname{pr}_{S}^{+}(r), \operatorname{pr}_{S}^{+}(r)\right\rangle_{Y}<0\right\} .
$$

This tessellation is the restriction to $\mathcal{P}_{Y}$ of the tessellation of $\mathcal{P}_{X}$ by induced chambers. Since $N(X)$ is a standard fundamental domain of the action of $W\left(S_{X}\right)$ on $\mathcal{P}_{X}$, Proposition 4.4 and $N(Y)=\mathcal{P}(Y) \cap N(X)$ imply the following.

Proposition 5.2 The chamber $N(Y)$ is tessellated by induced chambers.

Note that a chamber $D_{Y}$ in $\mathcal{P}_{Y}$ is an induced chamber if and only if there exists an induced chamber $D_{X}$ in $\mathcal{P}_{X}$ such that $D_{Y}=\mathcal{P}_{Y} \cap D_{X}$. More precisely, suppose that a subset $\mathcal{F}\left(D_{X}\right)$ of $S_{X}^{\vee} \backslash\{0\}$ defines an induced chamber $D_{X}$ in $\mathcal{P}_{X}$ :

$$
D_{X}=\left\{x \in \mathcal{P}_{X} \mid\langle v, x\rangle_{X} \geq 0 \text { for all } v \in \mathcal{F}\left(D_{X}\right)\right\} \text {. }
$$

Then we have $D_{X} \cap \mathcal{P}_{Y}=\left\{y \in \mathcal{P}_{Y} \mid\left\langle\operatorname{pr}^{+}(v), y\right\rangle_{Y} \geq 0\right.$ for all $\left.v \in \mathcal{F}\left(D_{X}\right)\right\}$. Hence, if $D_{X} \cap \mathcal{P}_{Y}$ contains a non-empty open subset of $\mathcal{P}_{Y}$, then $D_{X} \cap \mathcal{P}_{Y}$ is an induced chamber in $\mathcal{P}_{Y}$ defined by $\operatorname{pr}^{+}\left(\mathcal{F}\left(D_{X}\right)\right) \backslash\{0\}$. 
On an Enriques Surface Associated With a Quartic Hessian Surface

\subsection{Smooth Rational Curves on an Enriques Surface}

Let $X$ and $Y$ be as in the previous subsection. In particular, $S_{Y}$ is regarded as a $\mathbb{Z}$-submodule of $S_{X}$. Let $h_{Y} \in S_{Y}$ be an ample class of $Y$. Then $h_{Y} \in S_{X}$ is ample on $X$. We explain a method to calculate the finite subset

$$
\mathcal{R}_{d}:=\left\{[C] \in S_{Y} \mid C \text { is a smooth rational curve on } Y \text { such that }\left\langle[C], h_{Y}\right\rangle_{Y}=d\right\}
$$

of $S_{Y}$ for each positive integer $d$ by induction on $d$ starting from $\mathcal{R}_{0}=\varnothing$. Since the sublattice $S_{X}^{-}$of $S_{X}$ is negative-definite, the set $\mathcal{T}:=\left\{t \in S_{X}^{-} \mid\langle t, t\rangle_{X}=-4\right\}$ is finite and can be calculated. We have the following.

Lemma 5.3 (Nikulin [23]) Let $v \in S_{Y}$ be a vector such that $\langle v, v\rangle_{Y}=-2$ and $\left\langle v, h_{Y}\right\rangle_{Y}>0$. Then the following conditions are equivalent.

(i) The vector $v$ is the class of an effective divisor $D$ on $Y$ such that $\pi^{*} D$ is written as $[\Delta]+[\Delta]^{\varepsilon}$, where $\Delta$ is an effective divisor on $X$ satisfying $\left\langle[\Delta],[\Delta]^{\varepsilon}\right\rangle_{X}=0$.

(ii) There exists an element $t \in \mathcal{T}$ satisfying $(v+t) / 2 \in S_{X}$.

By the algorithm in $[26, \S 3]$, we compute the finite set

$$
\mathcal{V}_{d}:=\left\{v \in S_{Y} \mid\langle v, v\rangle_{Y}=-2,\left\langle v, h_{Y}\right\rangle_{Y}=d\right\} .
$$

We then compute $\mathcal{V}_{d}^{\prime}:=\left\{v \in \mathcal{V}_{d} \mid v\right.$ satisfies Lemma 5.3 (ii) $\}$. Lemma 5.3 implies that $\mathcal{R}_{d} \subset \mathcal{V}_{d}^{\prime}$.

Lemma 5.4 A vector $v$ in $\mathcal{V}_{d}^{\prime}$ fails to belong to $\mathcal{R}_{d}$ if and only if there exists a vector $r \in \mathcal{R}_{d^{\prime}}$ with $0<d^{\prime}<d$ such that $\langle v, r\rangle_{Y}<0$.

Proof Lemma 5.3 implies that there exists an effective divisor $D$ on $Y$ such that $v=$ $[D]$. Then $v \in \mathcal{R}_{d}$ if and only if $D$ is irreducible.

Suppose that there exists a smooth rational curve $C$ on $Y$ such that $\langle v,[C]\rangle_{Y}<0$. Then $D$ contains $C$. In particular, if $\left\langle[C], h_{Y}\right\rangle_{Y}<\left\langle v, h_{Y}\right\rangle_{Y}=d$, then $D$ is not irreducible and hence $v \notin \mathcal{R}_{d}$. Conversely, suppose that $D$ is reducible. Let $\Gamma_{1}, \ldots, \Gamma_{N}$ be the distinct reduced irreducible components of $D$. If $\left\langle[D],\left[\Gamma_{i}\right]\right\rangle_{Y} \geq 0$ for all $i$, we would have $\langle v, v\rangle_{Y} \geq 0$. Therefore there exists an irreducible component $\Gamma_{i}$ such that $\left\langle[D],\left[\Gamma_{i}\right]\right\rangle_{Y}<0$. Then we have $\left\langle\left[\Gamma_{i}\right],\left[\Gamma_{i}\right]\right\rangle_{Y}<0$ and hence $\Gamma_{i}$ is a smooth rational curve. Since $D$ is reducible, we see that $d^{\prime}:=\left\langle\left[\Gamma_{i}\right], h_{Y}\right\rangle_{Y}$ is smaller than $d$, and hence $r:=\left[\Gamma_{i}\right] \in \mathcal{R}_{d^{\prime}}$ satisfies $\langle v, r\rangle_{Y}<0$.

\subsection{An Elliptic Fibration on an Enriques Surface}

Let $Y$ be an Enriques surface with an ample class $h_{Y} \in S_{Y}$. Let $\phi: Y \rightarrow \mathbb{P}^{1}$ be an elliptic fibration. Then the class of a fiber of $\phi$ is written as $2 f_{\phi}$, where $f_{\phi}$ is primitive in $S_{Y}$. For $p \in \mathbb{P}^{1}$, let $E_{p}$ denote the divisor on $Y$ such that

$$
\phi^{-1}(p)= \begin{cases}E_{p} & \text { if } \phi^{-1}(p) \text { is not a multiple fiber } \\ 2 E_{p} & \text { if } \phi^{-1}(p) \text { is a multiple fiber. }\end{cases}
$$


We give a method to calculate the reducible fibers of $\phi$. Let $d_{\phi}:=\left\langle 2 f_{\phi}, h_{Y}\right\rangle_{Y}$ be the degree of a fiber of $\phi$ with respect to $h_{Y}$. Then we obviously have

$$
\begin{aligned}
\mathcal{R}(\phi):= & \left\{[C] \in S_{Y} \mid C \text { is a smooth rational curve on } Y \text { contained in a fiber of } \phi\right\} \\
& =\left\{[C] \in S_{Y} \mid[C] \in \mathcal{R}_{d} \text { for some } d<d_{\phi}, \text { and }\left\langle[C], f_{\phi}\right\rangle_{Y}=0\right\} .
\end{aligned}
$$

We calculate the dual graph of the roots in $\mathcal{R}(\phi)$, and decompose $\mathcal{R}(\phi)$ into equivalence classes according to the connected components of the graph. Then there exists a canonical bijection between the set of these equivalence classes and the set of reducible fibers of $\phi$. Let $\Gamma_{p} \subset \mathcal{R}(\phi)$ be one of the equivalence classes, and suppose that $\Gamma_{p}$ corresponds to a reducible fiber $\phi^{-1}(p)$. The roots in $\Gamma_{p}$ form an indecomposable extended Dynkin diagram, and its ADE-type is the ADE-type of the divisor $E_{p}$. We calculate $t\left(\Gamma_{p}\right):=\sum_{r \in \Gamma_{p}} m_{r} \cdot\left\langle r, h_{Y}\right\rangle_{Y}$, where $m_{r}$ is the multiplicity in $E_{p}$ of the irreducible component corresponding to $r \in \Gamma_{p}$ ( [9, Figure 1.8]). We have $t\left(\Gamma_{p}\right)=\left\langle\left[E_{p}\right], h_{Y}\right\rangle_{Y}$, and hence $t\left(\Gamma_{p}\right)$ is either $d_{\phi}$ or $d_{\phi} / 2$. Then $\phi^{-1}(p)$ is a multiple fiber if and only if $t\left(\Gamma_{p}\right)=d_{\phi} / 2$.

\section{General Quartic Hessian Surface}

We review the results on the general quartic Hessian surface obtained by Dolgachev and Keum [8], and re-calculate these results in the form of vectors and matrices. Henceforth, we denote by $X$ the minimal resolution of the general quartic Hessian surface $\bar{X}$ defined in Introduction.

It is known that the Néron-Severi lattice $S_{X}$ of $X$ is of rank 16, and $S_{X}^{\vee} / S_{X}$ is isomorphic to $(\mathbb{Z} / 2 \mathbb{Z})^{4} \times(\mathbb{Z} / 3 \mathbb{Z})$. If two lines $\ell_{\beta}$ and $\ell_{\beta^{\prime}}$ on $\bar{X}$ intersect, the intersection point is an ordinary node of $\bar{X}$. Hence we have

$$
\left\langle\left[E_{\alpha}\right],\left[E_{\alpha^{\prime}}\right]\right\rangle=(-2) \cdot \delta_{\alpha \alpha^{\prime}}, \quad\left\langle\left[L_{\beta}\right],\left[L_{\beta^{\prime}}\right]\right\rangle=(-2) \cdot \delta_{\beta \beta^{\prime}},
$$

where $\delta$ is Kronecker's delta symbol on $A \cup B$. Recall that the indexing of $p_{\alpha}$ and $\ell_{\beta}$ was done in such a way that the following holds:

$$
\left\langle\left[E_{\alpha}\right],\left[L_{\beta}\right]\right\rangle= \begin{cases}0, & \alpha \not \beta, \\ 1, & \alpha \supset \beta .\end{cases}
$$

The lattice $S_{X}$ is generated by the classes $\left[E_{\alpha}\right]$ and $\left[L_{\beta}\right]$. More precisely, the classes of the following smooth rational curves form a basis of $S_{X}$ :

$$
\begin{aligned}
& E_{123}, E_{124}, E_{125}, E_{134}, E_{135}, E_{145}, E_{234}, E_{235}, E_{245}, E_{345}, \\
& L_{45}, L_{35}, L_{34}, L_{25}, L_{24}, L_{13} .
\end{aligned}
$$

We fix this basis once and for all and write elements of $S_{X} \otimes \mathbb{R}$ as row vectors. The Gram matrix of $S_{X}$ with respect to this basis is readily calculated by (6.1) and (6.2). The fact that the classes of the 16 curves above form a basis of $S_{X}$ can be confirmed by checking that the determinant of this Gram matrix is equal to $-2^{4} \cdot 3$. Let $h_{Q} \in S_{X}$ denote the class of the pullback of a hyperplane section of $\bar{X} \subset \mathbb{P}^{3}$ by the minimal resolution $X \rightarrow \bar{X}$. Then we have $h_{Q}=[-1,1,1,-1,-1,1,1,1,3,1,2,0,0,2,2,-2]$. 


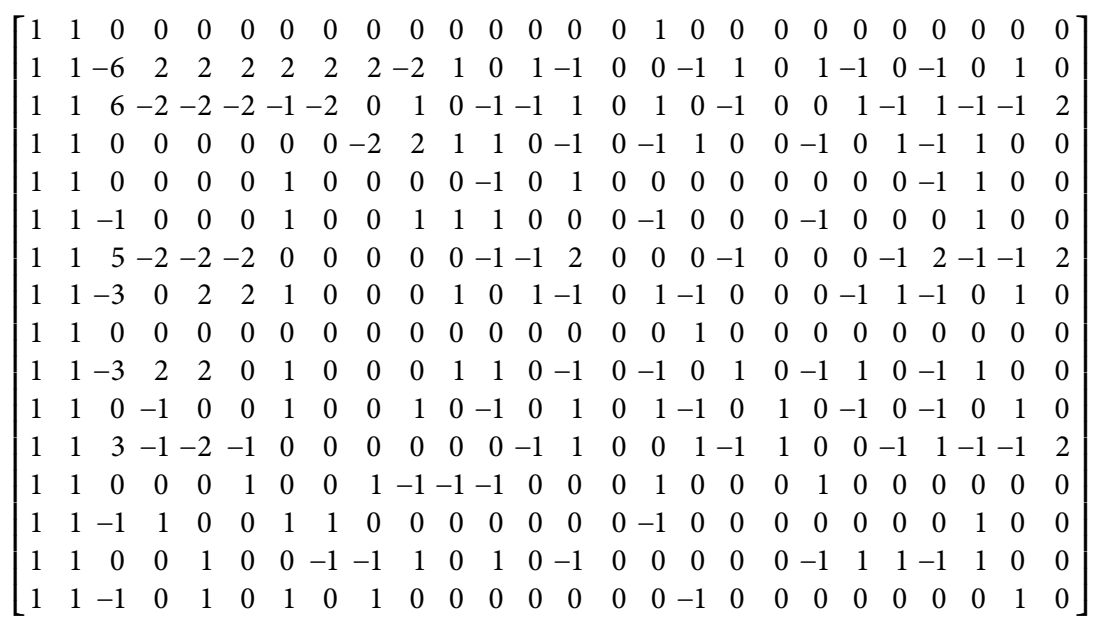

Table 6.1: Primitive embedding of $S_{X}$ into $L_{26}$.

Proposition 6.1 $\quad$ (i) An element $g$ of aut $(N(X))$ belongs to aut $(X)$ if and only if $g$ satisfies $\eta_{S_{X}}(g)= \pm 1$, where $\eta_{S_{X}}: \mathrm{O}\left(S_{X}\right) \rightarrow \mathrm{O}\left(q_{S_{X}}\right)$ is the natural homomorphism.

(ii) The representation $\varphi_{X}: \operatorname{Aut}(X) \rightarrow \operatorname{aut}(X)$ is an isomorphism.

Proof Since $\bar{X}$ is general in the family of quartic Hessian surfaces, we have

$$
\mathrm{O}^{\omega}\left(T_{X}\right)=\{ \pm 1\} .
$$

Therefore the statements follow from Theorem 4.7.

We then apply Borcherds's method to $S_{X}$. We fixed a basis $f_{1}, f_{2}, \lambda_{1}, \ldots, \lambda_{24}$ of $L_{26}$ in Section 3.2. Let $M$ be the matrix given in Table 6.1. It is easy see that the homomorphism $i: S_{X} \rightarrow L_{26}$ given by $v \mapsto v M$ is a primitive embedding of $S_{X}$ into $L_{26}$ that maps $\mathcal{P}_{X}$ into $\mathcal{P}_{26}$. Henceforth, we regard $S_{X}$ as a primitive sublattice of $L_{26}$ by this embedding $i$.

Remark 6.2 This embedding $i: S_{X} \rightarrow L_{26}$ is equal to the embedding given by Dolgachev and Keum [8] up to the action of $\mathrm{O}^{+}\left(S_{X}\right)$ and $\mathrm{O}^{+}\left(L_{26}\right)$. See [27] for a general method to embed the Néron-Severi lattice of a $K 3$ surface into $L_{26}$ in Borcherds's method.

As in Section 4.1, we denote the orthogonal projection by $\operatorname{pr}_{S}: L_{26} \otimes \mathbb{R} \rightarrow S_{X} \otimes \mathbb{R}$. We can calculate a Gram matrix of the orthogonal complement $R$ of $S_{X}$ in $L_{26}$ explicitly, and confirm that $R$ contains a root. Hence $R$ cannot be embedded into the negativedefinite Leech lattice $\Lambda$. Therefore Proposition 4.3 can be applied.

Let $D_{26}$ be the Conway chamber with the Weyl vector $w_{26}=f_{1} \in L_{26}$ (see Section 3.2). We put $D_{X}:=i^{-1}\left(D_{26}\right)$. 


\begin{tabular}{ccccc} 
type & $\left\langle v, h_{X}\right\rangle$ & $\langle v, v\rangle$ & number & \\
\hline (a) & 1 & -2 & 20 & outer \\
(b) & 2 & -1 & 10 & inner \\
(c) & 5 & $-2 / 3$ & 24 & inner \\
(d) & 4 & $-2 / 3$ & 30 & inner
\end{tabular}

Table 6.2: Walls of $D_{X}$

Proposition 6.3 The closed subset $D_{X}$ of $\mathcal{P}_{X}$ contains the vector

$$
h_{X}:=\operatorname{pr}_{S}\left(w_{26}\right)=[-3,2,2,-3,-3,2,2,2,7,2,5,0,0,5,5,-5]
$$

of square-norm 20 in its interior. Moreover, $h_{X}$ belongs to $N(X)$. Hence $D_{X}$ is an induced chamber contained in $N(X)$.

Proof We can calculate the set $\mathcal{F}^{\prime}\left(D_{X}\right):=\left\{\operatorname{pr}_{S}(r) \mid r \in \mathcal{W}_{26},\left\langle\operatorname{pr}_{S}(r), \operatorname{pr}_{S}(r)\right\rangle<0\right\}$ by the algorithm presented in $[27, \$ 5]$. By definition, we have

$$
D_{X}=\left\{x \in \mathcal{P}_{X} \mid\langle x, v\rangle \geq 0 \text { for all } v \in \mathcal{F}^{\prime}\left(D_{X}\right)\right\} .
$$

Hence we can confirm that $h_{X}$ is an interior point of $D_{X}$. Therefore $D_{X}$ is an induced chamber. Moreover, we can confirm that $\left\{r \in S_{X} \mid\left\langle r, h_{X}\right\rangle=0,\langle r, r\rangle=-2\right\}=\varnothing$ by the algorithm given in $[26, \$ 3]$. Since the singularities of $\bar{X}$ consist only of the ordinary nodes $p_{\alpha}(\alpha \in A)$, we have

$$
\left\{r \in S_{X} \mid\left\langle r, h_{Q}\right\rangle=0,\langle r, r\rangle=-2\right\}=\left\{ \pm\left[E_{\alpha}\right] \mid \alpha \in A\right\},
$$

and the classes $\left[E_{\alpha}\right]$ define the walls of $N(X)$ that pass through the half-line $\mathbb{R}_{\geq 0} h_{Q}$. We see that $\left\langle\left[E_{\alpha}\right], h_{X}\right\rangle=1>0$ for all $\alpha \in A$, and confirm that

$$
\left\{r \in S_{X} \mid\left\langle r, h_{Q}\right\rangle>0,\left\langle r, h_{X}\right\rangle<0,\langle r, r\rangle=-2\right\}=\varnothing
$$

by the algorithm given in $[26, \S 3]$. These facts imply that $h_{X}$ is an interior point of $N(X)$. By Proposition 4.4, $N(X)$ is tessellated by induced chambers. Hence $D_{X}$ is contained in $N(X)$.

Proposition 6.4 The number of walls of $D_{X}$ is $20+10+24+30=84$, among which 20 walls are walls of $N(X)$ and they are defined by the roots $\left[E_{\alpha}\right]$ and $\left[L_{\beta}\right]$, whereas the other $10+24+30$ walls are not walls of $N(X)$.

Proof In the proof of Proposition 6.3, the set $\mathcal{F}^{\prime}\left(D_{X}\right)$ of vectors defining the chamber $D_{X}$ was calculated. From $\mathcal{F}^{\prime}\left(D_{X}\right)$, we can calculate the set $\mathcal{F}\left(D_{X}\right)$ of primitive defining vectors $v$ of walls of $D_{X}$ by Algorithm 2.3. The result is given in Table 6.2. The walls of $D_{X}$ are divided into four types (a)-(d) according to the values of $\left\langle v, h_{X}\right\rangle$ and $\langle v, v\rangle$, where $v \in S_{X}^{\vee}$ is the primitive defining vector of the wall. It turns out that the 20 walls of type (a) are defined by $\left[E_{\alpha}\right]$ and $\left[L_{\beta}\right]$, and hence these walls are walls of $N(X)$. For each of the other walls, there exists no positive integer $k$ such that $k^{2}\langle v, v\rangle=-2$. Hence these walls are not walls of $N(X)$. 
We call a wall of $D_{X}$ an outer wall if it is a wall of $N(X)$, and call it an inner wall otherwise.

Proposition 6.5 If $g \in \operatorname{aut}(X)$, then $D_{X}^{g}$ is an induced chamber.

Proof Let $R$ denote the orthogonal complement of $S_{X}$ in $L_{26}$. By [22], we have an isomorphism $\sigma_{R}: q_{R} \stackrel{\sim}{\rightarrow}-q_{S_{X}}$ of finite quadratic forms given by the even unimodular overlattice $L_{26}$ of $S_{X} \oplus R$. Let $\sigma_{R *}: \mathrm{O}\left(q_{R}\right) \stackrel{\sim}{\rightarrow} \mathrm{O}\left(q_{s_{X}}\right)$ denote the isomorphism induced by $\sigma_{R}$. By Proposition 6.1, we have $\eta_{S_{X}}(g)= \pm 1$ and hence $\eta_{S_{X}}(g)$ belongs to the image of the composite homomorphism $\mathrm{O}(R) \stackrel{\eta_{R}}{\longrightarrow} \mathrm{O}\left(q_{R}\right) \stackrel{\sigma_{R *}}{\longrightarrow} \mathrm{O}\left(q_{s_{X}}\right)$. By [22] again, there exists an element $\widetilde{g}$ of $\mathrm{O}\left(L_{26}\right)$ such that $\widetilde{g}$ preserves the primitive sublattices $S_{X}$ and $R$ and that the restriction of $\widetilde{g}$ to $S_{X}$ is equal to $g$. Since $D_{X}^{g}=i^{-1}\left(D_{26}^{\widetilde{g}}\right)$, we see that $D_{X}^{g}$ is an induced chamber.

Proposition 6.6 The automorphism group aut $\left(D_{X}\right)$ of the chamber $D_{X}$ is of order 240 , and we have $h_{X}^{g}=h_{X}$ for all $g \in \operatorname{aut}\left(D_{X}\right)$.

Proof If $g \in \mathrm{O}^{+}\left(S_{X}\right)$ induces an automorphism of $D_{X}$, then $g$ induces a permutation of the set $\left\{\left(\left[E_{\alpha}\right]\right)^{\perp},\left(\left[L_{\beta}\right]\right)^{\perp}\right\}$ of walls of type $(\mathrm{a})$, and hence $g$ induces a permutation of $A \cup B$ that preserves the intersection numbers (6.1) and (6.2). The permutations of $A \cup B$ preserving (6.1) and (6.2) form a group of order 240 generated by the permutations of $\{1, \ldots, 5\}$ and the switch $\alpha=\bar{\beta} \leftrightarrow \beta=\bar{\alpha}$. Conversely, each of these 240 permutations induces an isometry of $S_{X}$ that preserves $D_{X}$. By direct calculation, we see that

$$
h_{X}=\sum_{\alpha \in A}\left[E_{\alpha}\right]+\sum_{\beta \in B}\left[L_{\beta}\right]
$$

Hence we have $h_{X}^{g}=h_{X}$ for all $g \in \operatorname{aut}\left(D_{X}\right)$.

Proposition 6.7 The group aut $\left(D_{X}\right) \cap \operatorname{aut}(X)$ is of order 2 and its non-trivial element $g_{\varepsilon}$ is the image $\varphi_{X}(\varepsilon)$ of an Enriques involution $\varepsilon \in \operatorname{Aut}(X)$ by the natural representation $\varphi_{X}: \operatorname{Aut}(X) \rightarrow \operatorname{aut}(X)$. This Enriques involution $\varepsilon$ switches $E_{\alpha}$ and $L_{\bar{\alpha}}$ for each $\alpha \in A$.

Proof By means of Proposition 6.1 (i), we can check by direct calculation that

$$
\operatorname{aut}\left(D_{X}\right) \cap \operatorname{aut}(X)
$$

consists of the identity and the isometry that comes from the switch $\alpha \leftrightarrow \bar{\alpha}$. The matrix presentation of the isometry $g_{\varepsilon} \in \operatorname{aut}\left(D_{X}\right)$ induced by the switch is given in Table 6.3. As in (5.1), we put

$$
S_{X}^{+}:=\left\{v \in S_{X} \mid v^{g_{\varepsilon}}=v\right\}, \quad S_{X}^{-}:=\left\{v \in S_{X} \mid v^{g_{\varepsilon}}=-v\right\} .
$$

Then $S_{X}^{+}$is of rank 10 generated by the row vectors $\eta_{1}, \ldots, \eta_{10}$ of the matrix given in Table 6.4. We see that $S_{X}^{+} \cong L_{10}(2)$. Indeed, we have chosen the basis $\eta_{1}, \ldots, \eta_{10}$ of $S_{X}^{+}$in such a way that the homomorphism from $L_{10}$ to $S_{X}^{+}$given by

$$
f_{1} \longmapsto \eta_{1}, \quad f_{2} \longmapsto \eta_{2}, \quad e_{1} \longmapsto \eta_{3}, \quad \ldots \quad e_{8} \longmapsto \eta_{10}
$$




$\left[\begin{array}{rrrrrrrrrrrrrrrr}0 & 0 & 0 & 0 & 0 & 0 & 0 & 0 & 0 & 0 & 1 & 0 & 0 & 0 & 0 & 0 \\ 0 & 0 & 0 & 0 & 0 & 0 & 0 & 0 & 0 & 0 & 0 & 1 & 0 & 0 & 0 & 0 \\ 0 & 0 & 0 & 0 & 0 & 0 & 0 & 0 & 0 & 0 & 0 & 0 & 1 & 0 & 0 & 0 \\ 0 & 0 & 0 & 0 & 0 & 0 & 0 & 0 & 0 & 0 & 0 & 0 & 0 & 1 & 0 & 0 \\ 0 & 0 & 0 & 0 & 0 & 0 & 0 & 0 & 0 & 0 & 0 & 0 & 0 & 0 & 1 & 0 \\ -2 & 1 & 1 & -2 & -2 & 1 & 0 & 0 & 3 & 0 & 2 & -1 & -1 & 2 & 2 & -3 \\ -1 & 1 & 0 & -1 & -2 & 0 & 1 & 0 & 2 & 0 & 1 & -1 & 0 & 1 & 2 & -2 \\ -1 & 0 & 1 & -2 & -1 & 0 & 0 & 1 & 2 & 0 & 1 & 0 & -1 & 2 & 1 & -2 \\ 0 & 0 & 0 & 0 & 0 & 0 & 0 & 0 & 0 & 0 & 0 & 0 & 0 & 0 & 0 & 1 \\ 0 & -1 & -1 & 1 & 1 & 0 & 0 & 0 & -1 & 1 & 0 & 1 & 1 & -1 & -1 & 1 \\ 1 & 0 & 0 & 0 & 0 & 0 & 0 & 0 & 0 & 0 & 0 & 0 & 0 & 0 & 0 & 0 \\ 0 & 1 & 0 & 0 & 0 & 0 & 0 & 0 & 0 & 0 & 0 & 0 & 0 & 0 & 0 & 0 \\ 0 & 0 & 1 & 0 & 0 & 0 & 0 & 0 & 0 & 0 & 0 & 0 & 0 & 0 & 0 & 0 \\ 0 & 0 & 0 & 1 & 0 & 0 & 0 & 0 & 0 & 0 & 0 & 0 & 0 & 0 & 0 & 0 \\ 0 & 0 & 0 & 0 & 1 & 0 & 0 & 0 & 0 & 0 & 0 & 0 & 0 & 0 & 0 & 0 \\ 0 & 0 & 0 & 0 & 0 & 0 & 0 & 0 & 1 & 0 & 0 & 0 & 0 & 0 & 0 & 0\end{array}\right]$

Table 6.3: Matrix representation of the Enriques involution $\varepsilon$.

$$
\left[\begin{array}{rrrrrrrrrrrrrrrr}
-1 & 1 & 0 & -1 & -1 & 1 & 1 & 0 & 2 & 1 & 2 & 0 & 0 & 1 & 2 & -2 \\
-1 & 1 & 1 & -1 & -1 & 0 & 1 & 1 & 2 & 0 & 1 & 0 & 0 & 2 & 2 & -2 \\
0 & 0 & 0 & 1 & 0 & 0 & 0 & 0 & 0 & 0 & 0 & 0 & 0 & 1 & 0 & 0 \\
-2 & 1 & 1 & -2 & -2 & 1 & 0 & 1 & 2 & 0 & 1 & 0 & -1 & 2 & 1 & -3 \\
1 & -1 & -1 & 2 & 1 & -1 & 1 & -1 & -2 & 1 & -1 & 0 & 2 & -2 & -1 & 2 \\
-1 & 1 & 1 & -3 & -2 & 1 & 0 & 1 & 3 & -1 & 2 & -1 & -2 & 2 & 2 & -3 \\
1 & -1 & -1 & 2 & 3 & -1 & -1 & 0 & -3 & 1 & -2 & 2 & 1 & -2 & -2 & 3 \\
1 & 0 & 0 & 1 & 0 & 0 & 0 & -1 & -1 & 0 & 0 & 0 & 1 & -1 & -1 & 1 \\
-2 & 1 & 1 & -2 & -2 & 1 & 1 & 0 & 3 & -1 & 1 & -2 & -1 & 2 & 3 & -3 \\
1 & 0 & -1 & 1 & 1 & -1 & 0 & 0 & -1 & 0 & -1 & 1 & 0 & -1 & -1 & 2
\end{array}\right]
$$

Table 6.4: Basis of $S_{X}^{+}$.

induces an isometry $L_{10}(2) \stackrel{\sim}{\rightarrow} S_{X}^{+}$, where $f_{1}, f_{2}, e_{1}, \ldots, e_{8}$ are the basis of $L_{10}$ fixed in Section 3.1. On the other hand, we can confirm that the negative-definite even lattice $S_{X}^{-}$has no roots (Proposition 7.11). Hence we conclude that $g_{\varepsilon}$ is the image $\varphi_{X}(\varepsilon)$ of an Enriques involution $\varepsilon$ on $X$ by the criterion given in [12].

The walls of $D_{X}$ of type (b) play an important role in the study of the Enriques surface $Y:=X /\langle\varepsilon\rangle$. For $\alpha \in A$, let $v_{\alpha}$ be the unique vector of $S_{X}^{\vee}$ that satisfies

$$
\left\langle v_{\alpha},\left[E_{\alpha^{\prime}}\right]\right\rangle=\delta_{\alpha \alpha^{\prime}}, \quad\left\langle v_{\alpha},\left[L_{\beta}\right]\right\rangle=\delta_{\bar{\alpha} \beta} .
$$

Then the walls of $D_{X}$ of type (b) are exactly the walls defined by $v_{\alpha}$ for some $\alpha \in A$. Since $g_{\varepsilon}$ comes from the switch $\alpha \leftrightarrow \bar{\alpha}$, we have $v_{\alpha}^{g_{\varepsilon}}=v_{\alpha}$.

Proposition 6.8 For $\alpha \in A$, let $\bar{\pi}_{\alpha}: \bar{X} \rightarrow \mathbb{P}^{2}$ be the projection from the center $p_{\alpha} \in$ $\bar{X}$, and let $\iota_{\alpha}: X \rightarrow X$ be the involution obtained from the double covering $\bar{\pi}_{\alpha}$. Then 
$g_{\alpha}:=\varphi_{X}\left(\iota_{\alpha}\right) \in \operatorname{aut}(X)$ maps $D_{X}$ to the induced chamber adjacent to $D_{X}$ across the wall $D_{X} \cap\left(v_{\alpha}\right)^{\perp}$ of type (b). Moreover $\iota_{\alpha}$ commutes with $\varepsilon$.

Proof Let $\pi_{\alpha}: X \rightarrow \mathbb{P}^{2}$ denote the composite of $\bar{\pi}_{\alpha}$ with the minimal resolution $X \rightarrow \bar{X}$. Then the class $\pi_{\alpha}^{*}([l]) \in S_{X}$ of the pullback of a line $l$ on $\mathbb{P}^{2}$ is $h_{Q}-\left[E_{\alpha}\right]$. We calculate the finite set

$$
\widetilde{\Gamma}_{\alpha}:=\left\{r \in S_{X} \mid\left\langle r, \pi_{\alpha}^{*}([l])\right\rangle=0,\langle r, r\rangle=-2,\left\langle r, h_{X}\right\rangle>0\right\}
$$

by the algorithm given in $[26, \S 3]$. From $\widetilde{\Gamma}_{\alpha}$, using the ample class $h_{X}$ of $X$, and by the method described in $[29, \$ 6.1]$ we can calculate the set $\Gamma_{\alpha}$ of classes of smooth rational curves that are contracted by $\pi_{\alpha}$. It turns out that the vectors in $\Gamma_{\alpha}$ form the following Dynkin diagram.

$$
\begin{array}{cccccccccccc}
E_{\alpha_{11}} & L_{\beta_{1}} & E_{\alpha_{12}} & E_{\alpha_{21}} & L_{\beta_{2}} & E_{\alpha_{22}} & E_{\alpha_{31}} & L_{\beta_{3}} & E_{\alpha_{32}} & E_{\alpha_{1}^{\prime}} & E_{\alpha_{2}^{\prime}} & E_{\alpha_{3}^{\prime}} \\
\circ & \circ & 0 & 0 & 0 & 0 & 0 & 0 & 0 & 0 & 0 & 0
\end{array}
$$

Here $\beta_{1}, \beta_{2}, \beta_{3}$ are the three elements of $B$ contained in $\alpha$, the three indices $\alpha, \alpha_{v 1}, \alpha_{v 2}$ are the three elements of $A$ containing $\beta_{v}$ for $v=1,2,3$, and $\alpha_{1}^{\prime}, \alpha_{2}^{\prime}, \alpha_{3}^{\prime}$ are the three elements of $A$ containing $\bar{\alpha} \in B$. In particular, the singular locus of the branch curve of $\bar{\pi}_{\alpha}: \bar{X} \rightarrow \mathbb{P}^{2}$ consists of 6 simple singular points, and its ADE-type is $3 A_{3}+3 A_{1}$. Then the eigenspace $V_{1}:=\operatorname{Ker}\left(g_{\alpha}-I_{16}\right)$ of $g_{\alpha}$ in $S_{X} \otimes \mathbb{Q}$ is of dimension 10 spanned by the classes

$$
h_{Q}-\left[E_{\alpha}\right], \quad\left[L_{\beta_{v}}\right], \quad\left[E_{\alpha_{v 1}}\right]+\left[E_{\alpha_{v 2}}\right], \quad\left[E_{\alpha_{\mu}^{\prime}}\right] \quad(v, \mu=1,2,3),
$$

and the eigenspace $V_{-1}:=\operatorname{Ker}\left(g_{\alpha}+I_{16}\right)$ is the orthogonal complement of $V_{1}$. Hence we can calculate the matrix representation of $g_{\alpha}$. See [31] for these matrices. We can confirm by direct calculation of products of matrices that $g_{\alpha}$ and $g_{\varepsilon}$ commute. Therefore $\varepsilon$ and $\iota_{\alpha}$ commute by Proposition 6.1 (ii). By Proposition 6.5, we know that $D_{X}^{g_{\alpha}}$ is an induced chamber. We can confirm that $v_{\alpha}^{g_{\alpha}}=-v_{\alpha}$, and that $\left\langle h_{X}^{g_{\alpha}}, v\right\rangle>0$ holds for all primitive defining vectors $v$ of walls of $D_{X}$ other than $v_{\alpha}$. Hence $D_{X}^{g_{\alpha}}$ is adjacent to $D_{X}$ across the inner wall $D_{X} \cap\left(v_{\alpha}\right)^{\perp}$ of $D_{X}$.

Dolgachev and Keum [8] also constructed automorphisms of $X$ whose action on $S_{X}$ maps $D_{X}$ to the induced chamber adjacent to $D_{X}$ across each inner wall of type (c) and type (d). Thus they obtained a set of generators of $\operatorname{Aut}(X)$. See [31] for the matrix representations of these automorphisms.

Lemma 6.9 Let $\sigma_{\alpha}$ and $\sigma_{\beta}$ denote the reflections of $S_{X}$ with respect to the roots $\left[E_{\alpha}\right]$ and $\left[L_{\beta}\right]$, respectively. Then $D_{X}^{\sigma_{\alpha}}$ and $D_{X}^{\sigma_{\beta}}$ are induced chambers.

Proof Since $\left[E_{\alpha}\right]$ and $\left[L_{\beta}\right]$ are roots of $L_{26}$, the reflections $\sigma_{\alpha}$ and $\sigma_{\beta}$ are the restrictions to $S_{X}$ of reflections of $L_{26}$.

Combining this fact with the automorphisms of $X$ constructed in [8], we obtain the following.

Corollary 6.10 The embedding $i: S_{X} \hookrightarrow L_{26}$ is of simple Borcherds type. 


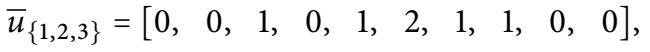

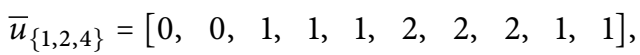

$$
\begin{aligned}
& \bar{u}_{\{1,2,5\}}=[0,1,-2,-1,-2,-3,-2,-1,-1,-1] \text {, } \\
& \bar{u}_{\{1,3,4\}}=\left[\begin{array}{lllllllll}
0, & 0, & 1, & 0, & 0, & 0, & 0, & 0, & 0,
\end{array}\right] \text {, } \\
& \bar{u}_{\{1,3,5\}}=\left[\begin{array}{llllllll}
0, & 0,1, & 0,1,2,2,1,1,0
\end{array}\right] \text {, } \\
& \bar{u}_{\{1,4,5\}}=[1,0,-2,-1,-3,-4,-3,-2,-1,-1] \text {, }
\end{aligned}
$$

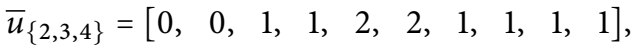

$$
\begin{aligned}
& \bar{u}_{\{2,3,5\}}=[0,1,-2,-1,-2,-3,-3,-3,-2,-1] \text {, } \\
& \bar{u}_{\{2,4,5\}}=[1,1,-5,-3,-6,-9,-7,-5,-3,-1] \text {, } \\
& \bar{u}_{\{3,4,5\}}=[1,0,-2,-1,-2,-4,-3,-3,-2,-1] \text {, } \\
& \bar{v}_{\{1,2,3\}}=\left[\begin{array}{lll}
0,1,-1, & 0,-1,-2,-1,-1,0,0
\end{array}\right] \text {, } \\
& \bar{v}_{\{1,2,4\}}=[1,1,-4,-3,-5,-8,-7,-5,-3,-2] \text {, } \\
& \bar{v}_{\{1,2,5\}}=[1,0,-1,-1,-2,-2,-2,-2,-1,0] \text {, } \\
& \bar{v}_{\{1,3,4\}}=\left[\begin{array}{lllllllll}
1, & 0,-1, & 0, & 0, & 0, & 0, & 0, & 0, & 0
\end{array}\right], \\
& \bar{v}_{\{1,3,5\}}=[1,1,-4,-2,-5,-8,-7,-5,-4,-2] \text {, } \\
& \bar{v}_{\{1,4,5\}}=\left[\begin{array}{ll}
0,1,-1,-1,-1,-2,-1,-1,-1, & 0
\end{array}\right] \text {, } \\
& \bar{v}_{\{2,3,4\}}=[1,1,-4,-3,-6,-8,-6,-5,-4,-2] \text {, } \\
& \bar{v}_{\{2,3,5\}}=[1,0,-1,-1,-1,-2,-1,0,0,0] \text {, } \\
& \bar{v}_{\{2,4,5\}}=\left[\begin{array}{llllllll}
0, & 0,2,2,3,4,3,2,1,0
\end{array}\right] \text {, } \\
& \bar{v}_{\{3,4,5\}}=[0,1,-1,-1,-2,-2,-2,-1,0,0]
\end{aligned}
$$

Table 7.1: Primitive defining vectors of the walls of $D_{Y}$.

\section{Geometry of the Enriques Surface $Y$}

Henceforth, we denote by $Y$ the quotient of the $K 3$ surface $X$ by the Enriques involution $\varepsilon$ given in Proposition 6.7.

\subsection{Chamber $D_{Y}$ and Generators of aut $(Y)$}

As in Section 5.1, we identify the $\mathbb{Z}$-module $S_{Y}$ with the $\mathbb{Z}$-submodule $S_{X}^{+}$of $S_{X}$ by $\pi^{*}$ so that we have $\mathcal{P}_{Y}=\left(S_{Y} \otimes \mathbb{R}\right) \cap \mathcal{P}_{X}$ and $N(Y)=N(X) \cap \mathcal{P}_{Y}$. We have fixed a basis $\eta_{1}, \ldots, \eta_{10}$ of $S_{X}^{+}$in such a way that the homomorphism (6.5) is an isometry $L_{10}(2) \stackrel{\sim}{\rightarrow} S_{X}^{+}$. We use $\eta_{1}, \ldots, \eta_{10}$ as a basis of $S_{Y}$, and write elements of $S_{Y} \otimes \mathbb{R}$ as row vectors. Hence the Gram matrix of $S_{Y}$ is equal to the standard Gram matrix of $L_{10}=U \oplus E_{8}$.

Recall that $S_{X}$ is embedded in $L_{26}$ by the matrix in Table 6.1. Let

$$
\operatorname{pr}_{S}^{+}: L_{26} \otimes \mathbb{R} \stackrel{\mathrm{pr}_{S}}{\rightarrow} S_{X} \otimes \mathbb{R} \stackrel{\mathrm{pr}^{+}}{\longrightarrow} S_{Y} \otimes \mathbb{R}
$$


denote the composite of the orthogonal projections $\mathrm{pr}_{S}$ and $\mathrm{pr}^{+}$. We consider the tessellation of $\mathcal{P}_{Y}$ given by the locally finite family of hyperplanes (5.2). We put

$$
D_{Y}:=\mathcal{P}_{Y} \cap D_{X} \text {. }
$$

By Proposition 6.6, we have $h_{X} \in S_{Y}$ and hence $h_{X} \in D_{Y}$. We put $h_{Y}:=h_{X}$. Note that the class $h_{Y} \in S_{Y}$ is ample on $Y$, and that $\left\langle h_{Y}, h_{Y}\right\rangle_{Y}=10$. With respect to the basis $\eta_{1}, \ldots, \eta_{10}$ of $S_{Y}$, we have $h_{Y}=[3,3,-8,-5,-10,-15,-12,-9,-6,-3]$.

Proposition 7.1 The closed subset $D_{Y}$ of $\mathcal{P}_{Y}$ is an induced chamber contained in $N(Y)$. The set of primitive defining vectors of walls of $D_{Y}$ consists of $10+10$ vectors

$$
\bar{u}_{\alpha}:=2 \operatorname{pr}^{+}\left(\left[E_{\alpha}\right]\right)=2 \operatorname{pr}^{+}\left(\left[L_{\bar{\alpha}}\right]\right), \quad \bar{v}_{\alpha}:=2 \operatorname{pr}^{+}\left(v_{\alpha}\right)
$$

of $S_{Y}^{\vee}$, where $\alpha$ runs through $A$, and $v_{\alpha} \in S_{X}^{\vee}$ is the primitive defining vector of an inner wall of $D_{X}$ of type (b), which is characterized by (6.6). The vector representations of $\bar{u}_{\alpha}$ and $\bar{v}_{\alpha}$ are given in Table 7.1.

Proof Since $h_{X}$ is an interior point of $D_{X}$ in $S_{X} \otimes \mathbb{R}$, the vector $h_{Y}=h_{X} \in D_{Y}$ is an interior point of $D_{Y}$ in $S_{Y} \otimes \mathbb{R}$. Hence $D_{Y}$ is a chamber. Since $D_{X}$ is an induced chamber contained in $N(X)$, the chamber $D_{Y}$ is an induced chamber contained in $N(Y)=\mathcal{P}_{Y} \cap N(X)$. By definition, the chamber $D_{Y}$ is defined by the set of vectors

$$
\left\{\operatorname{pr}^{+}(v) \mid v \in \mathcal{F}\left(D_{X}\right),\left\langle\operatorname{pr}^{+}(v), \operatorname{pr}^{+}(v)\right\rangle_{Y}<0\right\},
$$

where $\mathcal{F}\left(D_{X}\right)$ is the set of primitive defining vectors of walls of $D_{X}$, which we have calculated in the proof of Proposition 6.4. Using Algorithm 2.3, we obtain the set of primitive defining vectors of walls of $D_{Y}$ as Table 7.1.

Remark 7.2 If $v \in \mathcal{F}\left(D_{X}\right)$ defines a wall of $D_{X}$ of type (c) or (d), then we have $\left\langle\operatorname{pr}^{+}(v), \operatorname{pr}^{+}(v)\right\rangle_{Y}=0$, and hence the hyperplane $(v)^{\perp}$ of $\mathcal{P}_{X}$ does not intersect $\mathcal{P}_{Y} \subset \mathcal{P}_{X}$.

Corollary 7.3 The vector $\bar{u}_{\alpha}$ is the class of the smooth rational curve $\pi\left(E_{\alpha}\right)=\pi\left(L_{\bar{\alpha}}\right)$ on $Y$. In particular, each of the walls $D_{Y} \cap\left(\bar{u}_{\alpha}\right)^{\perp}$ is a wall of $N(Y)$.

The set of primitive defining vectors of walls of $D_{Y}$ is denoted by

$$
\mathcal{F}\left(D_{Y}\right):=\left\{\bar{u}_{\alpha} \mid \alpha \in A\right\} \cup\left\{\bar{v}_{\alpha} \mid \alpha \in A\right\} .
$$

We have

$$
\left\langle\bar{u}_{\alpha}, \bar{u}_{\alpha^{\prime}}\right\rangle_{Y}=\left\{\begin{aligned}
-2 & \text { if } \alpha=\alpha^{\prime} \\
1 & \text { if }\left|\alpha \cap \alpha^{\prime}\right|=1, \quad\left\langle\bar{v}_{\alpha}, \bar{v}_{\alpha^{\prime}}\right\rangle_{Y}=\left\{\begin{aligned}
-2 & \text { if } \alpha=\alpha^{\prime} \\
0 & \text { otherwise }
\end{aligned} \quad \text { if }\left|\alpha \cap \alpha^{\prime}\right|=2,\right. \\
0 & \text { otherwise }
\end{aligned}\right.
$$

and

$$
\left\langle\bar{u}_{\alpha}, \bar{v}_{\alpha^{\prime}}\right\rangle_{Y}=2 \delta_{\alpha \alpha^{\prime}} .
$$

Moreover, we have $\left\langle\bar{u}_{\alpha}, h_{Y}\right\rangle_{Y}=1$, and $\left\langle\bar{v}_{\alpha}, h_{Y}\right\rangle_{Y}=2$.

Proposition 7.4 If $\bar{g} \in \operatorname{aut}(Y)$, then $D_{Y}^{\bar{g}}$ is an induced chamber. 
Proof By Propositions 5.1 and 6.1, we have an element $g \in Z_{\text {aut }(X)}\left(g_{\varepsilon}\right)$ such that $\left.g\right|_{S_{Y}}=\bar{g}$. Since $D_{Y}^{\bar{g}}=\mathcal{P}_{Y} \cap D_{X}^{g}$, the statement follows from Proposition 6.5.

Proposition 7.5 The automorphism group aut $\left(D_{Y}\right)$ of the chamber $D_{Y}$ is isomorphic to the symmetric group of degree 5. Every element $\bar{g}$ of aut $\left(D_{Y}\right)$ satisfies $h_{Y}^{\bar{g}}=h_{Y}$. The intersection $\operatorname{aut}\left(D_{Y}\right) \cap \operatorname{aut}(Y)$ is trivial.

Proof Let $\bar{g}$ be an element of aut $\left(D_{Y}\right)$. Then $\bar{g}$ induces a permutation of $\mathcal{F}\left(D_{Y}\right)$ that preserves (7.1) and (7.2). Note that a vector $v \in \mathcal{F}\left(D_{Y}\right)$ belongs to $\left\{\bar{u}_{\alpha} \mid \alpha \in A\right\}$ (resp. to $\left.\left\{\bar{v}_{\alpha} \mid \alpha \in A\right\}\right)$ if there exist exactly three (resp. six) vectors $v^{\prime} \in \mathcal{F}\left(D_{Y}\right)$ such that $\left\langle v, v^{\prime}\right\rangle_{Y}=1$. Hence the permutation of $\mathcal{F}\left(D_{Y}\right)$ induced by $\bar{g}$ induces a permutation of $A$ preserving the set of pairs $\left\{\alpha, \alpha^{\prime}\right\}$ satisfying $\left|\alpha \cap \alpha^{\prime}\right|=1$. Therefore this permutation comes from a permutation of $\{1, \ldots, 5\}$. Conversely, it can easily be checked that a permutation of $\{1, \ldots, 5\}$ induces an isometry of $S_{Y}$. Hence the first assertion is proved. From (6.4), we have $h_{Y}=\sum_{\alpha \in A} \bar{u}_{\alpha}$. Hence the second assertion follows. Suppose that $\bar{g} \in \operatorname{aut}\left(D_{Y}\right)$ belongs to aut $(Y)$. By Propositions 5.1 and 6.1, there exists an element $g \in Z_{\text {aut }(X)}\left(g_{\varepsilon}\right)$ such that $\left.g\right|_{S_{Y}}=\bar{g}$. Since $h_{X}=h_{Y}$ and $h_{Y}^{\bar{g}}=h_{Y}$, we have $h_{X}^{g}=h_{X}$. Since $D_{X}^{g} \cap D_{X}$ contains an interior point $h_{X}$ of $D_{X}$, we see that $D_{X}^{g}=D_{X}$. By Proposition 6.7, it follows that $g \in\left\{\mathrm{id}, g_{\varepsilon}\right\}$. Therefore $\bar{g}$ is the identity.

The involution $\iota_{\alpha}: X \rightarrow X$ in Proposition 6.8 commutes with $\varepsilon$. Hence $\iota_{\alpha}$ induces an involution $j_{\alpha}: Y \rightarrow Y$ of $Y$, whose representation on $S_{Y}$ is $\bar{g}_{\alpha}:=g_{\alpha} \mid S_{Y}$, where $g_{\alpha} \in \operatorname{aut}(X)$ is the representation of $\iota_{\alpha}$ calculated in Proposition 6.8. We calculate the matrix representations of $\bar{g}_{\alpha}$. (See [31] for these matrices.)

Proposition 7.6 The element $\bar{g}_{\alpha} \in \operatorname{aut}(Y)$ maps $D_{Y}$ to the induced chamber adjacent to $D_{Y}$ across the wall $D_{Y} \cap\left(\bar{v}_{\alpha}\right)^{\perp}$.

Proof By Proposition 7.4, we know that $D_{Y}^{\bar{g}_{\alpha}}$ is an induced chamber. It is easy to check that $v_{\alpha} \in S_{X}^{+} \otimes \mathbb{R}$ and $v_{\alpha}^{g_{\alpha}}=-v_{\alpha}$, and hence $\bar{v}_{\alpha}^{\bar{g}_{\alpha}}=-\bar{v}_{\alpha}$. Therefore the vector $-\bar{v}_{\alpha}$ defines a wall of $D_{Y}^{\bar{g}_{\alpha}}$. Since $\left\langle h_{Y}^{\bar{g}_{\alpha}}, \bar{v}\right\rangle>0$ holds for all vectors $\bar{v}$ of $\mathcal{F}\left(D_{Y}\right) \backslash\left\{\bar{v}_{\alpha}\right\}$, we see that $D_{Y}^{\bar{g}_{\alpha}}$ is adjacent to $D_{Y}$ across the wall $D_{Y} \cap\left(\bar{v}_{\alpha}\right)^{\perp}$.

Remark 7.7 Each involution $\bar{g}_{\alpha}$ has an eigenvalue 1 with multiplicity 6 .

Proposition 7.8 For $\alpha \in A$, let $\bar{\sigma}_{\alpha} \in \mathrm{O}\left(S_{Y}\right)$ denote the reflection of $S_{Y}$ with respect to the root $\bar{u}_{\alpha}$. Then $\bar{\sigma}_{\alpha}$ maps $D_{Y}$ to the induced chamber adjacent to $D_{Y}$ across the wall $D_{Y} \cap\left(\bar{u}_{\alpha}\right)^{\perp}$.

Proof First remark that, since $S_{Y}$ is embedded in $L_{26}$ as $S_{Y}(2)$, the vector $\bar{u}_{\alpha}$ is not a root of $L_{26}$. Hence the argument of Lemma 6.9 does not work in this case. As was shown in Lemma 6.9, the chamber $D_{X}^{\sigma_{\alpha} \sigma_{\bar{\alpha}}}$ is an induced chamber in $\mathcal{P}_{X}$. By direct calculation, we see that $\sigma_{\alpha} \sigma_{\bar{\alpha}}$ commutes with $g_{\varepsilon}$ and that the restriction of $\sigma_{\alpha} \sigma_{\bar{\alpha}}$ to $S_{Y}$ is equal to $\bar{\sigma}_{\alpha}$. Hence $D_{Y}^{\bar{\sigma}_{\alpha}}$ is equal to $\mathcal{P}_{Y} \cap D_{X}^{\sigma_{\alpha} \sigma_{\bar{\alpha}}}$, and $D_{Y}^{\bar{\sigma}_{\alpha}}$ is an induced chamber. 
On an Enriques Surface Associated With a Quartic Hessian Surface

\begin{tabular}{lccccccccc}
$\operatorname{dim}$ & 1 & 2 & 3 & 4 & 5 & 6 & 7 & 8 & 9 \\
\hline outer faces & 657 & 3420 & 7250 & 8525 & 6270 & 2940 & 840 & 135 & 10 \\
aut $(Y)$-classes & 44 & 314 & 1077 & 1759 & 1669 & 1060 & 435 & 105 & 10 \\
\hline inner faces & 0 & 0 & 0 & 0 & 0 & 60 & 90 & 45 & 10 \\
$\operatorname{aut}(Y)$-classes & 0 & 0 & 0 & 0 & 0 & 1 & 15 & 25 & 10
\end{tabular}

Table 7.2: Numbers of faces of $D_{Y}$ and their aut $(Y)$-equivalence classes

Since $\bar{\sigma}_{\alpha}$ is the reflection in the hyperplane $\left(\bar{u}_{\alpha}\right)^{\perp}$, it is obvious that $D_{Y}^{\bar{\sigma}_{\alpha}}$ is adjacent to $D_{Y}$ across the wall $D_{Y} \cap\left(\bar{u}_{\alpha}\right)^{\perp}$.

From these propositions, we obtain the following corollaries.

Corollary 7.9 The primitive embedding $S_{Y}(2) \hookrightarrow S_{X} \hookrightarrow L_{26}$ of $S_{Y}(2)$ into $L_{26}$ is of simple Borcherds type.

Corollary 7.10 The group aut $(Y)$ is generated by the 10 involutions $\bar{g}_{\alpha}$. The induced chamber $D_{Y}$ is a fundamental domain of the action of $\operatorname{aut}(Y)$ on $N(Y)$. In particular, the mapping $\bar{g} \mapsto D_{Y}^{\bar{g}}$ gives rise to a bijection from aut $(Y)$ to the set of induced chambers contained in $N(Y)$.

\subsection{Smooth Rational Curves on $Y$}

Proposition 7.11 ( [16, Lemma 3.1]) The lattice $S_{X}^{-}$is isomorphic to $E_{6}(2)$, where $E_{6}$ is the negative-definite root lattice of type $E_{6}$.

Hence the set $\mathcal{T}$ of vectors of square-norm -4 in $S_{X}^{-}$consists of 72 elements. By the method in Section 5.2, we calculate the set $\mathcal{R}_{d}$ of the classes [C] of smooth rational curves $C$ on $Y$ with $\left\langle[C], h_{Y}\right\rangle_{Y}=d$ for $d=1, \ldots, 46$.

Proposition 7.12 Let $d$ be a positive integer $\leq 46$. If $d \not \equiv 1 \bmod 4$, then $\mathcal{R}_{d}$ is empty. If $d \equiv 1 \bmod 4$, then the cardinality of $\mathcal{R}_{d}$ is as follows.

\begin{tabular}{c|cccccccccccc}
$d$ & 1 & 5 & 9 & 13 & 17 & 21 & 25 & 29 & 33 & 37 & 41 & 45 \\
\hline$\left|\mathcal{R}_{d}\right|$ & 10 & 10 & 60 & 180 & 480 & 750 & 1440 & 2880 & 4110 & 5640 & 9480 & 11280
\end{tabular}

\subsection{Faces of $D_{Y}$ and Defining Relations of aut $(Y)$}

For the sake of readability, we will use the following notation:

$$
w(\alpha):=D_{Y} \cap\left(\bar{v}_{\alpha}\right)^{\perp}, \quad \bar{g}(\alpha):=\bar{g}_{\alpha} .
$$

We say that a face $F$ of $D_{Y}$ is inner if a general point of $F$ is an interior point of $N(Y)$, and that $F$ is outer otherwise. (An ideal face is obviously outer.) In particular, the walls $D_{Y} \cap\left(\bar{u}_{\alpha}\right)^{\perp}$ are outer, and $D_{Y} \cap\left(\bar{v}_{\alpha}\right)^{\perp}$ are inner. 
Definition 7.13 Let $F$ and $F^{\prime}$ be faces of $D_{Y}$. We put $F \sim F^{\prime}$ if there exists an element $\bar{g} \in \operatorname{aut}(Y)$ such that $F^{\prime}=F^{\bar{g}}$; that is, the induced chambers $D_{Y}$ and $D_{Y}^{\bar{g}}$ share the face $F^{\prime}$ and the face $F$ of $D_{Y}$ is mapped to the face $F^{\prime}$ of $D_{Y}^{\bar{g}}$ by $\bar{g}$. It is obvious that $\sim$ is an equivalence relation. When $F \sim F^{\prime}$, we say that $F$ and $F^{\prime}$ are aut $(Y)$-equivalent.

Proposition 7.14 The numbers of faces of $D_{Y}$ and their aut $(Y)$-equivalence classes are given in Table 7.2.

Proof The set of faces can be calculated from the set of walls of $D_{Y}$ by using Algorithm 2.3 iteratively. A face $F$ is outer if and only if there exists an outer wall of $D_{Y}$ containing $F$. Therefore we can make the lists of all outer faces and of all inner faces.

The set of aut $(Y)$-equivalence classes of faces is calculated by the following method. We put $F \stackrel{a}{\sim} F^{\prime}$ if there exists an inner wall $w(\alpha)$ of $D_{Y}$ containing the face $F$ such that $F=F^{\prime \bar{g}(\alpha)}$. (The superscript $a$ in the symbol $\stackrel{a}{\sim}$ is intended to mean "adjacent".) We show that the aut $(Y)$-equivalence relation $\sim$ is the smallest equivalence relation containing the relation $\stackrel{a}{\sim}$. Indeed, it is obvious that $F \stackrel{a}{\sim} F^{\prime}$ implies $F \sim F^{\prime}$. Suppose that $F \sim F^{\prime}$, and let $\bar{g} \in$ aut $(Y)$ be an element such that $F=F^{\prime \bar{g}}$. Looking at the tessellation of $N(Y)$ by induced chambers locally around a general point of $F$, we see that there exists a sequence of induced chambers $D_{0}=D_{Y}, D_{1}, \ldots, D_{m}=D_{Y}^{\bar{g}}$ with the following properties:

- Each $D_{i}$ contains $F$ and is contained in $N(Y)$.

- For $i=1, \ldots, m$, the induced chambers $D_{i-1}$ and $D_{i}$ are adjacent across a wall containing $F$.

Let $\bar{g}_{i}$ be an element of aut $(Y)$ such that $D_{i}=D_{Y}^{\bar{g}_{i}}$. Note that $\bar{g}_{i}$ is unique by Corollary 7.10. Let $w_{i}$ be the wall between $D_{i-1}$ and $D_{i}$. Since both of $D_{i-1}$ and $D_{i}$ are contained in $N(X)$, there exists an inner wall $w\left(\alpha_{i}\right)$ of $D_{Y}$ that is mapped to $w_{i}$ by $\bar{g}_{i-1}$. Then we have $\bar{g}_{i}=\bar{g}\left(\alpha_{i}\right) \bar{g}_{i-1}$, and hence $\bar{g}_{i}=\bar{g}\left(\alpha_{i}\right) \cdots \bar{g}\left(\alpha_{1}\right)$. Let $F_{i}$ be the face of $D_{Y}$ that is mapped to the face $F$ of $D_{i}$ by $\bar{g}_{i}$. Since $\bar{g}=\bar{g}_{m}$, we have $F_{m}=F^{\prime}$. Since $F_{i-1}=F_{i}^{\bar{g}\left(\alpha_{i}\right)}$, we have $F_{i-1} \stackrel{a}{\sim} F_{i}$. Therefore $F_{0}=F$ and $F_{m}=F^{\prime}$ are equivalent under the minimal equivalence relation containing $\stackrel{a}{\sim}$.

For each face $F$ of $D_{Y}$, we can make the finite list of all faces $F^{\prime}$ of $D_{Y}$ such that $F \stackrel{a}{\sim} F^{\prime}$. From these lists, we calculate the set of aut $(Y)$-equivalence classes of faces.

We give a description of inner faces of $D_{Y}$ of codimension 2. Let

$$
w(\alpha):=D_{Y} \cap\left(\bar{v}_{\alpha}\right)^{\perp}
$$

be an inner wall. For any $\alpha^{\prime} \in A \backslash\{\alpha\}$, the space $F_{\alpha^{\prime}}:=w(\alpha) \cap\left(\bar{v}_{\alpha^{\prime}}\right)^{\perp}$ contains a non-empty open subset of $\left(\bar{v}_{\alpha}\right)^{\perp} \cap\left(\bar{v}_{\alpha^{\prime}}\right)^{\perp}$. Indeed, the image $\operatorname{pr}\left(h_{Y}\right)$ of $h_{Y}$ by the orthogonal projection to $\left(\bar{v}_{\alpha}\right)^{\perp} \cap\left(\bar{v}_{\alpha^{\prime}}\right)^{\perp}$ satisfies $\left\langle\operatorname{pr}\left(h_{Y}\right), \bar{u}_{\alpha^{\prime \prime}}\right\rangle_{Y}>0$ for all $\alpha^{\prime \prime} \in A$ and $\left\langle\operatorname{pr}\left(h_{Y}\right), \bar{v}_{\alpha^{\prime \prime}}\right\rangle_{Y}>0$ for all $\alpha^{\prime \prime} \in A \backslash\left\{\alpha, \alpha^{\prime}\right\}$. Therefore the inner wall $w(\alpha)$ contains exactly 9 inner faces $F_{\alpha^{\prime}}$ of codimension 2. Let $x$ be a general point of $F_{\alpha^{\prime}}$. If $\left\langle\bar{v}_{\alpha}, \bar{v}_{\alpha^{\prime}}\right\rangle_{Y}=0$, then $\left(\bar{v}_{\alpha}\right)^{\perp}$ and $\left(\bar{v}_{\alpha^{\prime}}\right)^{\perp}$ intersect perpendicularly at $x$ and hence $x$ is contained in exactly 4 induced chambers of $N(Y)$, while, if $\left\langle\bar{v}_{\alpha}, \bar{v}_{\alpha^{\prime}}\right\rangle_{Y}=1$, then $\left(\bar{v}_{\alpha}\right)^{\perp}$ and $\left(\bar{v}_{\alpha^{\prime}}\right)^{\perp}$ intersect with angle $\pi / 3$ at $x$ and hence $x$ is contained in exactly 
6 induced chambers. These induced chambers lead to the relations among $\bar{g}(\alpha)$ in Proposition 7.16.

Proposition 7.15 Let $F$ be a non-ideal face of $D_{Y}$. Then the set

$$
\mathcal{G}(F):=\left\{\bar{g} \in \operatorname{aut}(Y) \mid F \subset D_{Y}^{\bar{g}}\right\}
$$

is finite and can be calculated explicitly.

Proof Note that the family of hyperplanes (5.2) that gives the tessellation of $\mathcal{P}_{Y}$ by induced chambers is locally finite in $\mathcal{P}_{Y}$. Since $F$ is not an ideal face, the number of induced chambers containing $F$ is finite. Hence $\mathcal{G}(F)$ is finite.

The set $\mathcal{G}(F)$ can be calculated as follows. We set $\mathrm{G}:=\{\mathrm{id}\}$, where id is the identity of $\operatorname{aut}(Y)$. Let $\mathrm{f}$ be the procedure that takes an element $\bar{g}$ of $\mathcal{G}(F)$ as an input and carries out the following task.

- Let $F^{\prime}$ be the face of $D_{Y}$ such that $F^{\prime \bar{g}}$ is equal to $F$. Calculate the set

$$
\left\{w\left(\alpha_{1}\right), \ldots, w\left(\alpha_{k}\right)\right\}
$$

of inner walls of $D_{Y}$ that contain $F^{\prime}$.

- For each $j=1, \ldots, k$, we calculate $\bar{g}^{\prime}:=\bar{g}\left(\alpha_{j}\right) \bar{g}$, which is an element of $\mathcal{G}(F)$, and if $\bar{g}^{\prime}$ is not yet in the set G, add $\bar{g}^{\prime}$ in $\mathrm{G}$ and input $\bar{g}^{\prime}$ to the procedure $f$.

We input id to the procedure $f$. It is easy to see that when the whole procedure terminates, the set $\mathrm{G}$ is equal to the set $\mathcal{G}(F)$.

Proposition 7.16 The following relations form a set of defining relations of aut $(Y)$ with respect to the set of generators $\{\bar{g}(\alpha) \mid \alpha \in A\} ; \bar{g}(\alpha)^{2}=$ id for each $\alpha \in A$,

$$
\left(\bar{g}(\alpha) \bar{g}\left(\alpha^{\prime}\right) \bar{g}\left(\alpha^{\prime \prime}\right)\right)^{2}=\mathrm{id}
$$

for each triple $\left(\alpha, \alpha^{\prime}, \alpha^{\prime \prime}\right)$ of distinct elements of $A$ such that $\left|\alpha \cap \alpha^{\prime} \cap \alpha^{\prime \prime}\right|=2$, and

$$
\left(\bar{g}(\alpha) \bar{g}\left(\alpha^{\prime}\right)\right)^{2}=\mathrm{id}
$$

for each pair $\left(\alpha, \alpha^{\prime}\right)$ such that $\left|\alpha \cap \alpha^{\prime}\right|=1$.

Proof By a standard argument of geometric group theory, there exists a one-to-one correspondence between a set of defining relations for aut $(Y)$, excluding $\bar{g}(\alpha)^{2}=\mathrm{id}$, and the set of 8-dimensional inner faces of $D_{Y}$. Let $F$ be an 8-dimensional inner face of $D_{Y}$. Then there exist exactly two walls of $D_{Y}$ containing $F$, and they are both inner. We put $D_{0}:=D_{Y}$ and choose an induced chamber $D_{1}$ from the two induced chambers that contain $F$ and are adjacent to $D_{0}$. Then there exists a cyclic sequence

$$
D_{0}, D_{1}, \ldots, D_{m-1}, D_{m}=D_{0}
$$

of induced chambers in $N(Y)$ with the following properties.

- For each $i \in \mathbb{Z} / m \mathbb{Z}, D_{i-1}$ and $D_{i+1}$ are the two induced chambers that contain $F$ and are adjacent to $D_{i}$.

- If $i, j \in \mathbb{Z} / m \mathbb{Z}$ are distinct, then $D_{i}$ and $D_{j}$ are distinct. 
We calculate the sequence of inner walls $w\left(\alpha_{1}\right), \ldots, w\left(\alpha_{m}\right)$ of $D_{Y}$ such that

$$
D_{i}=D_{Y}^{\bar{g}\left(\alpha_{i}\right) \cdots \bar{g}\left(\alpha_{1}\right)}
$$

Then we have $\bar{g}\left(\alpha_{m}\right) \cdots \bar{g}\left(\alpha_{1}\right)=\mathrm{id}$, and this is the defining relation corresponding to the inner face $F$. The cycle $D_{0}, D_{1}, \ldots, D_{m}$ for each $F$ can be computed by Proposition 7.15. Thus we obtain a list of defining relations from the list of inner faces of $D_{Y}$ that we calculated in Proposition 7.14.

The $45=10 \times 3+15$ inner faces of dimension 8 are decomposed into $25=10+15$ aut $(Y)$-equivalence classes. We see that if $w(\alpha)$ and $w\left(\alpha^{\prime}\right)$ are distinct inner walls, then $w(\alpha) \cap w\left(\alpha^{\prime}\right)$ is an 8-dimensional inner face. For each $\beta \in B$, there exist exactly three elements $\alpha, \alpha^{\prime}, \alpha^{\prime \prime} \in A$ that contain $\beta$, and the three 8-dimensional inner faces $w(\alpha) \cap w\left(\alpha^{\prime}\right), w\left(\alpha^{\prime}\right) \cap w\left(\alpha^{\prime \prime}\right)$, and $w\left(\alpha^{\prime \prime}\right) \cap w(\alpha)$ form an aut $(Y)$-equivalence class. The face $w(\alpha) \cap w\left(\alpha^{\prime}\right)$ corresponds to the relation

$$
\bar{g}(\alpha) \bar{g}\left(\alpha^{\prime \prime}\right) \bar{g}\left(\alpha^{\prime}\right) \bar{g}(\alpha) \bar{g}\left(\alpha^{\prime \prime}\right) \bar{g}\left(\alpha^{\prime}\right)=\mathrm{id}
$$

For each $i \in\{1, \ldots, 5\}$, there exist exactly three pairs $\left\{\alpha_{v}, \alpha_{v}^{\prime}\right\}(v=1,2,3)$ of elements of $A$ such that $\alpha_{v} \cap \alpha_{v}^{\prime}=\{i\}$. For each pair $\left\{\alpha_{v}, \alpha_{v}^{\prime}\right\}$, the 8-dimensional inner face $w\left(\alpha_{v}\right) \cap w\left(\alpha_{v}^{\prime}\right)$ forms an aut $(Y)$-equivalence class consisting of only one element. The face $w\left(\alpha_{v}\right) \cap w\left(\alpha_{v}^{\prime}\right)$ corresponds to the relation $\bar{g}\left(\alpha_{v}\right) \bar{g}\left(\alpha_{v}^{\prime}\right) \bar{g}\left(\alpha_{v}\right) \bar{g}\left(\alpha_{v}^{\prime}\right)=\mathrm{id}$. Thus Proposition 7.16 is proved.

\subsection{Proof of Theorem 1.1}

In [20], Enriques surfaces $Z$ with automorphisms that act on $S_{Z}$ trivially were classified. (See also $[14,19]$.) It follows that the action of $\operatorname{Aut}(Y)$ on $S_{Y}$ is faithful. Then Theorem 1.1 follows immediately from Corollary 7.10 and Proposition 7.16.

\subsection{Elliptic Fibrations of $Y$}

We prove Theorem 1.4. The $657=57+600$ one-dimensional faces of $D_{Y}$ are divided into $44=21+23$ aut $(Y)$-equivalence classes. Among them, there exist exactly 57 ideal faces, and they are divided into 21 aut $(Y)$-equivalence classes.

Let $\phi: Y \rightarrow \mathbb{P}^{1}$ be an elliptic fibration, and let $2 f_{\phi} \in S_{Y}$ denote the class of a fiber of $\phi$. There exists an isometry $g \in \operatorname{aut}(Y)$ that maps $f_{\phi}$ in an ideal face of $D_{Y}$. Conversely, let $F$ be an ideal face of $D_{Y}$, and let $f \in S_{Y}$ be the primitive vector such that $F=\mathbb{R}_{\geq 0} f$. Since $f$ is nef and satisfies $\langle f, f\rangle_{Y}=0$, there exists an elliptic fibration $\phi: Y \rightarrow \mathbb{P}^{1}$ such that $f=f_{\phi}$. Therefore there exists a bijection between the set of elliptic fibrations modulo the action of $\operatorname{Aut}(Y)$ and the set of aut $(Y)$-equivalence classes of ideal faces of $D_{Y}$.

For each ideal face $F=\mathbb{R}_{\geq 0} f$ with $f \in S_{Y}$ primitive, the ADE-type of reducible fibers of the corresponding elliptic fibration can be calculated from $f$, the ample class $h_{Y}$, and the sets $\mathcal{R}_{d}$ calculated in Proposition 7.12 by the method described in Section 5.3. Thus we obtain Table 1.1 and hence Theorem 1.4 is proved. 


\subsection{RDP-configurations on $Y$}

We prove Theorem 1.5. Let $\psi: Y \rightarrow \bar{Y}$ be a birational morphism to a surface $\bar{Y}$ that has only rational double points as its singularities, and let $h_{\psi} \in S_{Y}$ be the pullback of the class of a hyperplane section of $\bar{Y}$. Composing $\psi$ with an automorphism of $Y$, we assume that $h_{\psi} \in D_{Y}$. We see that the set

$$
\mathcal{R}(\psi):=\left\{[C] \in S_{Y} \mid C \text { is a smooth rational curve on } Y \text { contracted by } \psi\right\}
$$

can be calculated from the face $F$ of $D_{Y}$ that contains $h_{\psi}$ in its interior. Indeed, since $\left\langle h_{\psi}, h_{\psi}\right\rangle_{Y}>0$, the face $F$ is not an ideal face, and hence we can calculate the set $\mathcal{G}(F)$ defined in Proposition 7.15. Then $\mathcal{R}(\psi)$ is equal to

$\left\{\bar{u}_{\alpha}^{\bar{g}} \mid \bar{g}\right.$ is an element of $\mathcal{G}(F)$ such that the wall $\left(D_{Y} \cap\left(\bar{u}_{\alpha}\right)^{\perp}\right)^{\bar{g}}$ of $D_{Y}^{\bar{g}}$ contains $\left.F\right\}$.

Therefore we write $\mathcal{R}(\psi)$ as $\mathcal{R}(F)$. Conversely, let $F$ be a non-ideal face of $D_{Y}$, and let $h_{F}$ be an element of $F \cap S_{Y}$ that is not contained in any wall of $F$. Multiplying $h_{F}$ by a positive integer if necessary, we can assume that the line bundle $L_{F} \rightarrow Y$ corresponding to $h_{F}$ is globally generated and defines a morphism $\Phi_{\left|L_{F}\right|}: Y \rightarrow \mathbb{P}^{m}$. Let $Y \stackrel{\psi}{\rightarrow} \bar{Y} \rightarrow \mathbb{P}^{m}$ be the Stein factorization of $\Phi_{\left|L_{F}\right|}$. Then we have $\mathcal{R}(\psi)=\mathcal{R}(F)$.

We calculate $\mathcal{R}(F)$ for all non-ideal faces of $D_{Y}$. For two non-ideal faces $F$ and $F^{\prime}$, we put $F \leq F^{\prime}$ if $F$ is a face of $F^{\prime}$ and $\mathcal{R}(F)=\mathcal{R}\left(F^{\prime}\right)$ holds. Looking at the maximal faces with respect to this partial ordering, and dividing them into aut $(Y)$-equivalence classes, we obtain Table 1.2 and hence Theorem 1.5 is proved.

\subsection{Vinberg Chambers in $D_{Y}$}

In this subsection, we identify $S_{Y}$ with $L_{10}$ by the isometry $L_{10} \stackrel{\sim}{\rightarrow} S_{Y}$ given by (6.5). In particular, the chamber $D_{Y}$ is contained in $\mathcal{P}_{10}$. Note that the primitive defining vectors $\bar{u}_{\alpha}$ and $\bar{v}_{\alpha}$ of walls of $D_{Y}$ are roots (see (7.1)), and hence $D_{Y}$ is tessellated by Vinberg chambers.

Proof of Theorem 1.7. Let $V_{0}$ denote the Vinberg chamber $D_{10}$; see Section 3.1. We put $\varepsilon_{i}\left(V_{0}\right):=e_{i}$ for $i=1, \ldots, 10$, and let $m_{i}\left(V_{0}\right)$ denote the wall $V_{0} \cap\left(\varepsilon_{i}\left(V_{0}\right)\right)^{\perp}$ of $V_{0}$. Then, for each $i=1, \ldots, 9$, there exists a unique non-ideal one-dimensional face $F_{i}\left(V_{0}\right)$ of $V_{0}$ that is not contained in the wall $m_{i}\left(V_{0}\right)$. We denote by $f_{i}\left(V_{0}\right) \in L_{10}$ the primitive vector such that $F_{i}\left(V_{0}\right)=\mathbb{R}_{\geq 0} f_{i}\left(V_{0}\right)$. Then we have $f_{1}\left(V_{0}\right)=h_{Y}$ under the identification $L_{10}=S_{Y}$. (In fact, we have chosen the isomorphism (6.5) in such a way that $f_{1}\left(V_{0}\right)=h_{Y}$ holds.)

Let $V$ be an arbitrary Vinberg chamber in $\mathcal{P}_{10}$. Since the automorphism group $\operatorname{aut}\left(V_{0}\right)$ of $V_{0}$ is trivial, there exists a unique isometry $\bar{g}(V) \in \mathrm{O}^{+}\left(L_{10}\right)$ that maps $V_{0}$ to $V$. We put

$$
\varepsilon_{i}(V):=\varepsilon_{i}\left(V_{0}\right)^{\bar{g}(V)}, \quad m_{i}(V):=m_{i}\left(V_{0}\right)^{\bar{g}(V)}, \quad f_{i}(V):=f_{i}\left(V_{0}\right)^{\bar{g}(V)} .
$$

We say that a primitive vector $v$ of $L_{10}$ is an $f_{1}$-vector if $v=f_{1}(V)$ for some Vinberg chamber $V$. Let $v=f_{1}(V)$ be an $f_{1}$-vector. We put

$$
S(v):=\left\{V^{\prime} \mid V^{\prime} \text { is a Vinberg chamber such that } v=f_{1}\left(V^{\prime}\right)\right\} .
$$


Since the defining roots $\varepsilon_{2}(V), \ldots, \varepsilon_{10}(V)$ of the walls $m_{2}(V), \ldots, m_{10}(V)$ of $V$ containing $f_{1}(V)$ form a Dynkin diagram of type $A_{9}$, the cardinality of $S(v)$ is equal to $\left|\mathfrak{S}_{10}\right|$. We then put $\Sigma(v):=\bigcup_{V^{\prime} \in S(v)} V^{\prime}$, and call it a $\Sigma$-chamber with the center $v$. It is obvious that $\mathcal{P}_{10}$ is tessellated by $\sum$-chambers. The defining roots $\varepsilon_{2}(V), \varepsilon_{3}(V)$, $\varepsilon_{5}(V), \ldots, \varepsilon_{10}(V)$ of the walls of $V$ that contain $f_{1}(V)$ and are perpendicular to the wall $m_{1}(V)$ opposite to $f_{1}(V)$ form a Dynkin diagram of type $A_{2}+A_{6}$. Hence there exist exactly $\left|\mathfrak{S}_{3} \times \mathfrak{S}_{7}\right|$ Vinberg chambers $V^{\prime}$ in $S(v)$ such that $m_{1}(V)$ and $m_{1}\left(V^{\prime}\right)$ are supported on the same hyperplane. Hence the number of walls of the chamber $\Sigma(v)$ is

$$
\frac{\left|\mathfrak{S}_{10}\right|}{\left|\mathfrak{S}_{3} \times \mathfrak{S}_{7}\right|}=\frac{10 !}{3 ! \times 7 !}=120 .
$$

In particular, we see that the number of $\Sigma$-chambers that are adjacent to the $\Sigma$-chamber $\Sigma(v)$ is 120. Moreover, we can calculate the list $\left\{v_{1}, \ldots, v_{120}\right\}$ of centers of these adjacent $\Sigma$-chambers.

Let $v$ be an $f_{1}$-vector. If $v$ belongs to the interior of $D_{Y}$, then all 10 ! Vinberg chambers contained in $\Sigma(v)$ are contained in $D_{Y}$. If $v$ does not belong to $D_{Y}$, then none of the Vinberg chambers in $\Sigma(v)$ is contained in $D_{Y}$. Suppose that $v$ is located on the boundary of $D_{Y}$. We calculate the Dynkin diagram $\Delta$ formed by the roots

$$
\left\{\bar{u}_{\alpha} \mid\left\langle\bar{u}_{\alpha}, v\right\rangle_{Y}=0\right\} \cup\left\{\bar{v}_{\alpha} \mid\left\langle\bar{v}_{\alpha}, v\right\rangle_{Y}=0\right\}
$$

that define walls of $D_{Y}$ containing $v$. This Dynkin diagram $\Delta$ is a sub-diagram of the Dynkin diagram of type $A_{9}$. Let $W(\Delta) \subset \mathfrak{S}_{10}$ denote the corresponding subgroup. Then, among the 10 ! Vinberg chambers in $\Sigma(v)$, exactly $\left|\mathfrak{S}_{10}\right| /|W(\Delta)|$ Vinberg chambers are contained in $D_{Y}$.

Starting from the $f_{1}$-vector $h_{Y}$, we cover $D_{Y}$ by $\Sigma$-chambers, and count the number of Vinberg chambers in $D_{Y}$. By this method, Theorem 1.7 is proved.

Recall that $Z_{\text {gen }}$ is a generic Enriques surface. The natural representation

$$
\operatorname{Aut}\left(Z_{\text {gen }}\right) \rightarrow \mathrm{O}^{+}\left(L_{10}\right)
$$

is injective by [2]. Let aut $\left(Z_{\text {gen }}\right)$ denote the image of this homomorphism. Let aut ${ }^{\prime}(Y)$ denote the subgroup of $\mathrm{O}^{+}\left(L_{10}\right)$ generated by aut $(Y)$ and the ten reflections $\bar{\sigma}_{\alpha}$ with respect to the roots $\bar{u}_{\alpha} \in S_{Y}=L_{10}$.

Theorem 7.17 (i) The group $\operatorname{aut}^{\prime}(Y)$ contains aut $\left(Z_{\text {gen }}\right)$ as a normal subgroup, and $\operatorname{aut}^{\prime}(Y) / \operatorname{aut}\left(Z_{\text {gen }}\right)$ is isomorphic to the Weyl group $W\left(E_{6}\right)$ of type $E_{6}$.

(ii) The induced chamber $D_{Y}$ is a fundamental domain of the action of aut ${ }^{\prime}(Y)$ on $\mathcal{P}_{Y}$.

Proof Recall that the natural homomorphism $\rho: \mathrm{O}^{+}\left(L_{10}\right) \rightarrow \mathrm{O}\left(q_{L_{10}(2)}\right) \cong \mathrm{GO}_{10}^{+}(2)$ is surjective. By [2], we know that $\operatorname{aut}\left(Z_{\text {gen }}\right)$ is equal to $\operatorname{Ker} \rho$, and hence

$$
\left[\mathrm{O}^{+}\left(L_{10}\right): \operatorname{aut}\left(Z_{\text {gen }}\right)\right]=46998591897600=51840 \cdot 906608640 .
$$

By the brute force method using [10], we see that the order of the subgroup of $\mathrm{GO}_{10}^{+}(2)$ generated by $10+10$ elements $\rho\left(\bar{\sigma}_{\alpha}\right)$ and $\rho\left(\bar{g}_{\alpha}\right)$ is 51840 . Hence we have

$$
\left[\operatorname{aut}^{\prime}(Y): \operatorname{aut}^{\prime}(Y) \cap \operatorname{aut}\left(Z_{\text {gen }}\right)\right]=51840 .
$$


On the other hand, by Propositions 7.4, 7.8, and Theorem 1.7, we have

$$
\left[\mathrm{O}^{+}\left(L_{10}\right): \operatorname{aut}^{\prime}(Y)\right]=\frac{906608640}{\left|\operatorname{aut}^{\prime}(Y) \cap \operatorname{aut}\left(D_{Y}\right)\right|} .
$$

Hence we obtain $\left|\operatorname{aut}^{\prime}(Y) \cap \operatorname{aut}\left(D_{Y}\right)\right|=1$, which implies the assertion (ii). Moreover, we have $\operatorname{aut}\left(Z_{\text {gen }}\right) \triangleleft \operatorname{aut}^{\prime}(Y)$ and $\left|\operatorname{aut}^{\prime}(Y) / \operatorname{aut}\left(Z_{\text {gen }}\right)\right|=51840$.

In the following, we denote by $D(L)$ the discriminant group $L^{\vee} / L$ of an even lattice $L$, by $q(L)$ the discriminant form of $L$, and by $\eta(L): \mathrm{O}(L) \rightarrow \mathrm{O}(q(L))$ the natural homomorphism. We have $\left|D\left(S_{X}\right)\right|=2^{4} \cdot 3,\left|D\left(S_{X}^{+}\right)\right|=2^{10}$, and by Proposition 7.11, $\left|D\left(S_{X}^{-}\right)\right|=2^{6} \cdot 3$. By [22], the even overlattice $S_{X}$ of $S_{X}^{+} \oplus S_{X}^{-}$defines an isotropic subgroup $H_{S}:=S_{X} /\left(S_{X}^{+} \oplus S_{X}^{-}\right) \subset D\left(S_{X}^{+}\right) \oplus D\left(S_{X}^{-}\right)$such that

$$
H_{S} \cap D\left(S_{X}^{+}\right)=0, \quad H_{S} \cap D\left(S_{X}^{-}\right)=0, \quad\left|H_{S}^{\perp} / H_{S}\right|=2^{4} \cdot 3 .
$$

Hence we have $\left|H_{S}\right|=2^{6}$. Let $\left(D\left(S_{X}^{-}\right)_{2}, q\left(S_{X}^{-}\right)_{2}\right)$ denote the 2-part of

$$
\left(D\left(S_{X}^{-}\right), q\left(S_{X}^{-}\right)\right) \cong\left(D\left(E_{6}(2)\right), q\left(E_{6}(2)\right)\right),
$$

which can be regarded as a quadratic form of Witt defect 1 on $\mathbb{F}_{2}^{6}$. The automorphism group $\mathrm{GO}_{6}^{-}(2)$ of this quadratic form is isomorphic to $W\left(E_{6}\right)$. (See [1, p. 26].) Since $\left|H_{S}\right|=\left|D\left(S_{X}^{-}\right)_{2}\right|=2^{6}$, the second projection induces an isomorphism $\gamma_{H}: H_{S} \stackrel{\sim}{\rightarrow}$ $D\left(S_{X}^{-}\right)_{2}$, and the composite of $\gamma_{H}^{-1}$ and the first projection defines an embedding

$$
\gamma:\left(D\left(E_{6}(2)\right), q\left(E_{6}(2)\right)\right) \cong\left(D\left(S_{X}^{-}\right)_{2}, q\left(S_{X}^{-}\right)_{2}\right) \longleftrightarrow\left(D\left(S_{X}^{+}\right), q\left(S_{X}^{+}\right)\right) .
$$

Note that the image $H$ of $\gamma$ is equal to the image of the natural homomorphism $S_{X} \rightarrow$ $S_{X}^{+\vee} \rightarrow D\left(S_{X}^{+}\right)$. Let $q_{H}$ denote the restriction of $q\left(S_{X}^{+}\right)$to $H$, and $q_{H^{\perp}}$ the restriction to the orthogonal complement $H^{\perp}$ of $H$ in $\left(D\left(S_{X}^{+}\right), q\left(S_{X}^{+}\right)\right)$. Since $q_{H}$ is isomorphic to $-q\left(S_{X}^{-}\right)_{2}$, we have $\mathrm{O}\left(q_{H}\right) \cong \mathrm{GO}_{6}^{-}(2) \cong W\left(E_{6}\right)$. We consider the homomorphism

$$
\rho^{\prime}: \operatorname{aut}^{\prime}(Y) \longleftrightarrow \mathrm{O}^{+}\left(S_{Y}\right) \cong \mathrm{O}^{+}\left(S_{X}^{+}\right) \stackrel{\eta\left(S_{X}^{+}\right)}{\longrightarrow} \mathrm{O}\left(q\left(S_{X}^{+}\right)\right) .
$$

Since the homomorphism $\mathrm{O}^{+}\left(S_{Y}\right) \cong \mathrm{O}^{+}\left(S_{X}^{+}\right) \rightarrow \mathrm{O}\left(q\left(S_{X}^{+}\right)\right)$is identified with $\rho$, the homomorphism $\rho^{\prime}$ embeds aut ${ }^{\prime}(Y) /$ aut $\left(Z_{\text {gen }}\right)$ into $\mathrm{O}\left(q\left(S_{X}^{+}\right)\right)$. Every element of the image of $\operatorname{aut}^{\prime}(Y) \hookrightarrow \mathrm{O}^{+}\left(S_{Y}\right) \cong \mathrm{O}^{+}\left(S_{X}^{+}\right)$lifts to an element of $\mathrm{O}\left(S_{X}\right)$. Indeed, $\bar{g}_{\alpha} \epsilon$ $\operatorname{aut}^{\prime}(Y)$ lifts to $g_{\alpha} \in \mathrm{O}\left(S_{X}\right)$, and $\bar{\sigma}_{\alpha} \in$ aut $^{\prime}(Y)$ lifts to $\sigma_{\alpha} \sigma_{\bar{\alpha}} \in \mathrm{O}\left(S_{X}\right)$ (see the proof of Proposition 7.8). Hence every element in the image of $\rho^{\prime}$ preserves the factors $H$ and $H^{\perp}$ of $D\left(S_{X}^{+}\right)=H \oplus H^{\perp}$. By direct computation, we see that $\rho^{\prime}\left(\bar{g}_{\alpha}\right)$ and $\rho^{\prime}\left(\bar{\sigma}_{\alpha}\right)$ act on $H^{\perp}$ trivially. Therefore $\operatorname{aut}^{\prime}(Y) / \operatorname{aut}\left(Z_{\text {gen }}\right)$ can be regarded as a subgroup of $\mathrm{O}\left(q_{H}\right)$. Comparing the order, we obtain $\operatorname{aut}^{\prime}(Y) / \operatorname{aut}\left(Z_{\text {gen }}\right) \cong \mathrm{O}\left(q_{H}\right) \cong W\left(E_{6}\right)$.

Remark 7.18 Note that the lift $\sigma_{\alpha} \sigma_{\bar{\alpha}} \in \mathrm{O}^{+}\left(S_{X}\right)$ of $\bar{\sigma}_{\alpha} \in \operatorname{aut}^{\prime}(Y)$ satisfies the period condition $\eta_{S_{X}}\left(\sigma_{\alpha} \sigma_{\bar{\alpha}}\right) \in\{ \pm 1\}$ (see Proposition 6.1). By the specialization of $Z_{\text {gen }}$ to $Y$, the period condition is weakened and aut $\left(Z_{\text {gen }}\right)$ becomes the larger group aut ${ }^{\prime}(Y)$ with $10+10$ generators $\bar{g}_{\alpha}$ and $\bar{\sigma}_{\alpha}$. The presence of smooth rational curves on $Y$, however, prevents the 10 generators $\bar{\sigma}_{\alpha}$ from entering into aut $(Y)$. 


\section{Entropy}

Recently, many works have appeared on the distribution of entropies $\log \lambda(g)$ of automorphisms $g$ of $K 3$ or Enriques surfaces, where $\lambda(g)$ is the spectral radius of the action of $g$ on the Néron-Severi lattice of the surface. In particular, the problem of determining the minimum of the positive entropies in a certain class of automorphisms has been studied, for example, in $[7,18,24]$. In this section, we report the result of a computational experiment on the entropies of automorphisms of $Z_{\text {gen }}$.

From [2] and Theorem 7.17 we have the following equalities:

$$
\begin{aligned}
\operatorname{aut}\left(Z_{\text {gen }}\right) & =\operatorname{Ker}\left(\rho: \mathrm{O}^{+}\left(L_{10}\right) \rightarrow \mathrm{GO}_{10}^{+}(2)\right) \\
& =\operatorname{Ker}\left(\left.\rho\right|_{\operatorname{aut}^{\prime}(Y)}: \operatorname{aut}^{\prime}(Y) \rightarrow \mathrm{GO}_{6}^{-}(2)\right) .
\end{aligned}
$$

Since we know finite sets of generators for $\mathrm{O}^{+}\left(L_{10}\right)$ and for aut ${ }^{\prime}(Y)$, we can obtain a finite set of generators of aut $\left(Z_{\mathrm{gen}}\right)$ by the Reidemeister-Schreier method [17, Chapter 2]) from each of these descriptions of aut $\left(Z_{\mathrm{gen}}\right)$. Since $\left|\mathrm{GO}_{10}^{+}(2)\right|$ is very large, however, making use of the first equality is not practical. On the other hand, since $\left|\mathrm{GO}_{6}^{-}(2)\right|$ is much smaller compared with $\left|\mathrm{GO}_{10}^{+}(2)\right|$, we have managed to obtain a finite set of generators of aut $\left(Z_{\text {gen }}\right)$ in a reasonable computation time by means of the second equality.

Using this generating set, we search for elements $g \in \operatorname{aut}\left(Z_{\text {gen }}\right)$ with small $\lambda(g)$ for each degree $d=2,4, \ldots, 10$ of the minimal polynomial $s_{\lambda(g)}$ of the Salem number $\lambda(g)$. Below is the list of the smallest values of $\lambda(g)$ among the ones we found by an extensive random search of elements of aut $\left(Z_{\text {gen }}\right)$. See [31] for the matrices $g$ with these spectral radii.

\begin{tabular}{clc}
$d$ & $s_{\lambda(g)}$ & $\lambda(g)$ \\
\hline 2 & $t^{2}-14 t+\cdots$ & $13.9282 \cdots$ \\
4 & $t^{4}-16 t^{3}+14 t^{2}-\cdots$ & $15.1450 \cdots$ \\
6 & $t^{6}-38 t^{5}-49 t^{4}-84 t^{3}-\cdots$ & $39.3019 \cdots$ \\
8 & $t^{8}-68 t^{7}+68 t^{6}-188 t^{5}+118 t^{4}-\cdots$ & $67.0269 \cdots$ \\
10 & $t^{10}-138 t^{9}-19 t^{8}-248 t^{7}+18 t^{6}-252 t^{5}+\cdots$ & $138.1505 \cdots$
\end{tabular}

Remark 8.1 The famous Lehmer's number $\lambda_{\text {Leh }}=1.17628 \cdots$ is the spectral radius of a Coxeter element $c$ of $\mathrm{O}^{+}\left(L_{10}\right)=W\left(L_{10}\right)$. The order of $\rho(c) \in \mathrm{GO}_{10}^{+}(2)$ is 31 , and the spectral radius of $c^{31} \in \operatorname{aut}\left(Z_{\mathrm{gen}}\right)$ is equal to $\lambda_{\mathrm{Leh}}^{31}=153.4056 \cdots$.

Acknowledgements Thanks are due to I. Dolgachev, S. Kondo, S. Mukai, V. Nikulin, K. Oguiso, H. Ohashi, and M. Schütt for many discussions. In particular, Professor Kondo suggested the isomorphism $\operatorname{aut}^{\prime}(Y) /$ aut $\left(Z_{\text {gen }}\right) \cong W\left(E_{6}\right)$ in Theorem 7.17. The author also thanks the referee for many valuable comments on the first version of this paper. 


\section{References}

[1] J. H. Conway, R. T. Curtis, S. P. Norton, R. A. Parker, and R. A. Wilson, Atlas of finite groups. Oxford University Press, Eynsham, 1985.

[2] W. Barth and C. Peters, Automorphisms of Enriques surfaces. Invent. Math. 73(1983), no. 3 , 383-411. http://dx.doi.org/10.1007/BF01388435

[3] Richard Borcherds, Automorphism groups of Lorentzian lattices. J. Algebra 111(1987), no. 1, 133-153, 1987. http://dx.doi.org/10.1016/0021-8693(87)90245-6

[4] Coxeter groups, Lorentzian lattices, and K3 surfaces. Internat. Math. Res. Notices no.19, 1011-1031, 1998. http://dx.doi.org/10.1155/S1073792898000609

[5] J. H. Conway, The automorphism group of the 26-dimensional even unimodular Lorentzian lattice. J. Algebra 80(1983), no. 1, 159-163. http://dx.doi.org/10.1016/0021-8693(83)90025-X

[6] Elisa Dardanelli and Bert van Geemen, Hessians and the moduli space of cubic surfaces. In: Algebraic geometry. Contemp. Math., 422. Amer. Math. Soc., Providence, RI, 2007, pp. 17-36. http://dx.doi.org/10.1090/conm/422/08054

[7] Igor Dolgachev, Salem numbers and Enriques surfaces. Exp. Math. 27(2018), 287-301. http://dx.doi.org/10.1080/10586458.2016.1261743

[8] Igor Dolgachev and Jonghae Keum, Birational automorphisms of quartic Hessian surfaces. Trans. Amer. Math. Soc. 354(2002), no. 8, 3031-3057. http://dx.doi.org/10.1090/S0002-9947-02-03011-8

[9] Wolfgang Ebeling, Lattices and codes. Advanced Lectures in Mathematics. Third edition. Springer Spektrum, Wiesbaden 2013. http://dx.doi.org/10.1007/978-3-658-00360-9

[10] The GAP Group, GAP - Groups, Algorithms, and Programming. Version 4.7.9; 2015 http://www.gap-system.org.

[11] Toshiyuki Katsura, Shigeyuki Kondo, and Ichiro Shimada, On the supersingular K3 surface in characteristic 5 with Artin invariant 1. Michigan Math. J. 63(2014), no. 4, 803-844. http://dx.doi.org/10.1307/mmj/1417799227

[12] Jonghae Keum, Every algebraic Kummer surface is the K3-cover of an Enriques surface. Nagoya Math. J. 118(1990), 99-110. http://dx.doi.org/10.1017/S0027763000003019

[13] Kenji Koike, Hessian K3 surfaces of non-Sylvester type. J. Algebra 330(2011), 388-403. http://dx.doi.org/10.1016/j.jalgebra.2010.12.006

[14] Shigeyuki Kondō, Enriques surfaces with finite automorphism groups. Japan. J. Math. (N.S.) 12(1986), no. 2, 191-282. http://dx.doi.org/10.4099/math1924.12.191

[15] — The automorphism group of a generic Jacobian Kummer surface. J. Algebraic Geom. 7(1998), no. 3, 589-609.

[16] — The moduli space of Hessian quartic surfaces and automorphic forms. J. Pure Appl. Algebra 216(2012), no. 10, 2233-2240. http://dx.doi.org/10.1016/j.jpaa.2012.02.001

[17] Wilhelm Magnus, Abraham Karrass, and Donald Solitar, Combinatorial group theory. Second edition. Dover Publications, Mineola, NY, 2004.

[18] Curtis T. McMullen, Automorphisms of projective K3 surfaces with minimum entropy. Invent. Math. 203(2016), no. 1, 179-215.

[19] Shigeru Mukai, Numerically trivial involutions of Kummer type of an Enriques surface. Kyoto J. Math. 50(2010), no. 4, 889-902. http://dx.doi.org/10.1215/0023608X-2010-017

[20] Shigeru Mukai and Yukihiko Namikawa, Automorphisms of Enriques surfaces which act trivially on the cohomology groups. Invent. Math. 77(1984), no. 3, 383-397. http://dx.doi.org/10.1007/BF01388829

[21] S. Mukai and H. Ohashi, The automorphism groups of Enriques surfaces covered by symmetric quartic surfaces. In: Recent advances in algebraic geometry. London Math. Soc. Lecture Note Ser., 417. Cambridge Univ. Press, Cambridge, 2015, pp. 307-320.

[22] V. V. Nikulin, Integer symmetric bilinear forms and some of their geometric applications. Izv. Akad. Nauk SSSR Ser. Mat. 43(1979), no. 1, 111-177, 238.

[23] — Description of automorphism groups of Enriques surfaces. Dokl. Akad. Nauk SSSR 277(1984), no. 6, 1324-1327.

[24] Keiji Oguiso, The third smallest Salem number in automorphisms of K3 surfaces. Adv. Stud. Pure Math., 60. Math. Soc. Japan, Tokyo, 2010, pp. 331-360. http://dx.doi.org/10.1142/e031

[25] I. I. Piatetski-Shapiro and I. R. Shafarevich, Torelli's theorem for algebraic surfaces of type K3. Izv. Akad. Nauk SSSR Ser. Mat. 35(1971), 530-572. Reprinted in I. R. Shafarevich, Collected Mathematical Papers, Springer-Verlag, Berlin, 1989, pp. 516-557.

[26] Ichiro Shimada, Projective models of the supersingular K3 surface with Artin invariant 1 in characteristic 5. J. Algebra 403(2014), 273-299. http://dx.doi.org/10.1016/j.jalgebra.2013.12.029 
[27] — An algorithm to compute automorphism groups of K3 surfaces and an application to singular K3 surfaces. Int. Math. Res. Not. IMRN (2015), no. 22, 11961-12014.

[28] $\longrightarrow$ Holes of the Leech lattice and the projective models of K3 surfaces. Math. Proc. Cambridge Philos. Soc. 163(2017), 125-143. http://dx.doi.org/10.1017/S030500411600075X

[29] — The automorphism groups of certain singular K3 surfaces and an Enriques surface. In: K3 surfaces and their moduli. Progr. Math., 315. Birkhäuser/Springer, Basel, 2016, pp. 297-343. http://dx.doi.org/10.1007/978-3-319-29959-4_12

[30] Rational double points on Enriques surfaces, 2017. arxiv:1710.01461

[31] $\longrightarrow$ On an Enriques surface associated with a quartic Hessian surface: computational data 2016. http://www.math.sci.hiroshima-u.ac.jp/ shimada/K3andEnriques.html

[32] È. B. Vinberg. Some arithmetical discrete groups in Lobačevskil spaces. In: Discrete subgroups of Lie groups and applications to moduli. Oxford Univ. Press, Bombay, 1975, pp. 323-348.

Division of Mathematics, Graduate School of Science, Hiroshima University, 1-3-1 Kagamiyama, HigashiHiroshima, 739-8526, Japan

Email: ichiro-shimada@hiroshima-u.ac.jp 